\title{
Tissue-specific transcriptomics and proteomics of a filarial nematode and its Wolbachia endosymbiont
}

Ashley N. Luck', Kathryn G. Anderson², Colleen M. McClung³, Nathan C. VerBerkmoes³, Jeremy M. Foster', Michelle L. Michalski ${ }^{2+}$ and Barton E. Slatko ${ }^{1 *+}$

\begin{abstract}
Background: Filarial nematodes cause debilitating human diseases. While treatable, recent evidence suggests drug resistance is developing, necessitating the development of novel targets and new treatment options. Although transcriptomic and proteomic studies around the nematode life cycle have greatly enhanced our knowledge, whole organism approaches have not provided spatial resolution of gene expression, which can be gained by examining individual tissues. Generally, due to their small size, tissue dissection of human-infecting filarial nematodes remains extremely challenging. However, canine heartworm disease is caused by a closely related and much larger filarial nematode, Dirofilaria immitis. As with many other filarial nematodes, D. immitis contains Wolbachia, an obligate bacterial endosymbiont present in the hypodermis and developing oocytes within the uterus. Here, we describe the first concurrent tissue-specific transcriptomic and proteomic profiling of a filarial nematode (D. immitis) and its Wolbachia $(w D i)$ in order to better understand tissue functions and identify tissue-specific antigens that may be used for the development of new diagnostic and therapeutic tools.
\end{abstract}

Methods: Adult D. immitis worms were dissected into female body wall (FBW), female uterus (FU), female intestine $(\mathrm{FI})$, female head (FH), male body wall (MBW), male testis (MT), male intestine (MI), male head (MH) and 10.1186/ s12864-015-2083-2 male spicule (MS) and used to prepare transcriptomic and proteomic libraries.

Results: Transcriptomic and proteomic analysis of several D. immitis tissues identified many biological functions enriched within certain tissues. Hierarchical clustering of the D. immitis tissue transcriptomes, along with the recently published whole-worm adult male and female D. immitis transcriptomes, revealed that the whole-worm transcriptome is typically dominated by transcripts originating from reproductive tissue. The uterus appeared to have the most variable transcriptome, possibly due to age. Although many functions are shared between the reproductive tissues, the most significant differences in gene expression were observed between the uterus and testis. Interestingly, wDi gene expression in the male and female body wall is fairly similar, yet slightly different to that of Wolbachia gene expression in the uterus. Proteomic methods verified $32 \%$ of the predicted D. immitis proteome, including over 700 hypothetical proteins of $D$. immitis. Of note, hypothetical proteins were among some of the most abundant Wolbachia proteins identified, which may fulfill some important yet still uncharacterized biological function.

Conclusions: The spatial resolution gained from this parallel transcriptomic and proteomic analysis adds to our understanding of filarial biology and serves as a resource with which to develop future therapeutic strategies against filarial nematodes and their Wolbachia endosymbionts.

Keywords: Nematode, Filaria, Transcriptomics, Proteomics, Endosymbiosis, Wolbachia

\footnotetext{
* Correspondence: slatko@neb.com

${ }^{\dagger}$ Equal contributors

'Genome Biology Division, New England Biolabs, Inc., 240 County Road,

Ipswich, MA 01938, USA

Full list of author information is available at the end of the article
}

\section{Biomed Central}

(c) 2015 Luck et al. Open Access This article is distributed under the terms of the Creative Commons Attribution 4.0 International License (http://creativecommons.org/licenses/by/4.0/), which permits unrestricted use, distribution, and reproduction in any medium, provided you give appropriate credit to the original author(s) and the source, provide a link to the Creative Commons license, and indicate if changes were made. The Creative Commons Public Domain Dedication waiver (http://creativecommons.org/publicdomain/zero/1.0/) applies to the data made available in this article, unless otherwise stated. 


\section{Background}

Afflicting nearly 150 million people, filarial nematodes responsible for lymphatic filariasis (Wuchereria bancrofti, Brugia malayi and B. timori) and onchocerciasis (Onchocerca volvulus) are a worldwide source of disablement and morbidity [1]. Although current microfilaricidal treatments (which kill the larval stages) disrupt transmission of these insect vector-borne diseases [2], treatment is contraindicated in certain regions due to adverse drug reactions in the presence of another filarial nematode, Loa loa. Additionally, because recent evidence suggests drug resistance in filarial nematodes is developing [3, 4], the identification of novel vaccine targets and the development of new treatment options are necessary.

Many filarial nematode species (including W. bancrofti, B. malayi, B. timori and O. volvulus) contain Wolbachia, an obligate intracellular endosymbiotic bacterium found in the lateral cords of all adult worms as well as within the oocytes and developing embryos in the female reproductive tract [5-7]. Depletion of Wolbachia through tetracycline antibiotic treatment leads to decreased fertility and eventually death of adult worms [8-10]. Genome sequencing of filarial nematodes and their Wolbachia endobacteria has identified a number of critical metabolites implicated in the host-endosymbiont interaction [11-13]. For example, most filarial nematodes lack genes necessary for the synthesis of critical cofactors such as heme and riboflavin, as well as the de novo synthesis of purines and pyrimidines while these pathways are complete and likely functional within their corresponding Wolbachia genomes $[11,14]$. On the other hand, Wolbachia only partially maintains biosynthetic pathways for the synthesis of Coenzyme A, NAD, biotin, lipoic acid, ubiquinone and pyridoxal phosphate and thus, these cofactors may be supplied to the bacterium by the nematode host $[11,14]$.

While increasingly more filarial nematode genome sequences (and their respective Wolbachia counterparts) are becoming readily available, other 'omics' approaches (transcriptomics/proteomics) are needed to provide more detailed functional information. Previous transcriptomics (RNA-seq) studies have analyzed gene expression throughout the filarial nematode life cycle $[15,16]$. Nevertheless, these whole organism approaches do not provide spatial resolution of gene expression, which can be gained by tissue specific gene expression profiling. Given the relatively small size of many filarial nematodes (B. malayi adults typically range from 13 to $55 \mathrm{~mm}$ in length), tissue dissection is extremely challenging. Hence, information on tissuespecific gene expression in nematodes has been limited to the widely studied free-living nematode Caenorhabditis elegans and more recently Ascaris suum, a non-filarial nematode parasite [17]. While many filarial nematodes are relatively small in size, Dirofilaria immitis, the causative agent of canine heartworm disease, is much larger in both length $(20-30 \mathrm{~cm})$ and diameter, enabling more accurate dissection of tissues. Here, we describe the first tissue specific transcriptomic profiling of a filarial nematode (D. immitis) and its Wolbachia endosymbiont. Although RNA levels may imply potential translated protein levels, transcriptomics alone cannot provide any direct information about protein abundance in a tissue. Therefore, we concurrently performed mass spectrometry-based proteome analysis on a number of $D$. immitis tissues in order to corroborate transcriptomic data, expand our understanding of filarial nematode biology and identify tissue specific proteins that may be of interest in filarial nematode drug, diagnostic and vaccine development.

\section{Methods}

\section{D. immitis tissue preparation}

Adult $D$. immitis were obtained from the NIH/NIAID Filariasis Research Reagent Resource (FR3) Center for mass spectrometry analysis or purchased from TRS Labs for transcriptomic analysis. All animal procedures were conducted in accordance with IACUC guidelines. Biological replicates for transcriptomic analysis were from two untreated laboratory derived D. immitis-infected dogs. Worm dissections were performed using a dissecting microscope on either live or frozen adult $D$. immitis by mounting them on a wax pad and making a long head-to-tail incision using iridectomy scissors. The identity of each tissue piece removed from the worms was confirmed microscopically before tissues were rinsed three times in PBS prior to freezing at $-80{ }^{\circ} \mathrm{C}$. Worms were dissected into the following tissues or regions of the body: female body wall (FBW), female uterus (FU), female intestine (FI), female head (FH), male body wall $(\mathrm{MBW})$, male testis (MT), male intestine (MI), male head $(\mathrm{MH})$ and male spicule (MS). The D. immitis uterus tissue consists of the entire female reproductive tract, including the intrauterine contents (oocytes, developing embryos and immature microfilariae) and the male testis tissue comprised the entire male reproductive tract including sperm. Likewise, both the D. immitis male and female intestine preparations contain the entire nematode digestive tract posterior to the pharynx. The worm head was isolated by making a cut approximately $1 \mathrm{~mm}$ posterior to the nerve ring as visualized via stereomicroscope. The female and male head tissues consisted of the entire head including cuticle, body wall, mouth, amphidial neurons, nerve ring with some peripheral nerves as well as the pharynx. Male spicule samples represent the male tale severed from the body approximately $1 \mathrm{~mm}$ anterior of the spicule shafts. All D. immitis tissues were fast frozen in $1.5 \mathrm{~mL}$ graduated round 
bottom tubes (Eppendorf) and stored at $-80{ }^{\circ} \mathrm{C}$ prior to RNA or protein isolation.

\section{Total RNA isolation and library preparation}

Tissue samples were homogenized with ceramic beads in CK14 tubes using a Minilys homogenizer (Precellys) and total RNA was extracted by organic extraction using Trizol (Ambion). Samples were treated with DNase I (Ambion) at $37{ }^{\circ} \mathrm{C}$ for 30 min before further Trizol extraction and final purification. The RNA integrity, purity and concentration of all samples were assessed on an RNA nano chip using a Bioanalyzer 2100 (Agilent Technologies). In order to capture any Wolbachia transcripts, total RNA was used to prepare all RNA-Seq libraries. Samples were prepared for sequencing using the NEBNext ${ }^{\circ}$ mRNA Library Prep Master Mix Set for Illumina ${ }^{\circ}$ (New England Biolabs), according to the instructions. Library quality was assessed using a DNA high sensitivity chip on a Bioanalyzer 2100 .

\section{Transcriptome sequencing and bioinformatic analysis}

Transcriptomic libraries were sequenced using an Illumina GAIIx sequencer. Data were collected for $50 \mathrm{bp}$ single-end reads. All data was analyzed using a local instance of Galaxy [18-20]. Sequence reads from each tissue sample were first assessed for quality using FastQC ( $\mathrm{v}$ 1.0.0) [21] and further analyzed using the Tuxedo protocol [22]. RNA-Seq reads from each sample were aligned to the D. immitis genome (version 2.2) [14] using TopHat (v. 1.4.1) [23]. Default parameters were used except for the maximum number of alignments to be allowed was set to 40. Reads aligned using TopHat were assembled into transcripts using Cufflinks (v. 1.3.0). Default parameters were used. Cufflinks assemblies from all samples were merged using Cuffmerge (v.1.0.0) and used for differential expression testing using Cuffdiff (v.1.3.0), with the false discovery rate (FDR) set to 0.01 .

Similarly, the RNA-Seq reads from each sample were also mapped to the Wolbachia from D. immitis ( $w \mathrm{Di}$ ) genome (version 2.2) [14] using Bowtie [24]. Reads aligned using Bowtie were assembled into transcripts using Cufflinks, then merged with Cuffmerge. Differential expression profiles were determined using Cuffdiff (v. 2.1.1). Default parameters for Cuffdiff were used except the minimum alignment count was set to 2 and FDR set to 0.01 .

Hierarchical clustering analysis was performed using Cluster 3.0 [25]. Mapped reads from biological replicates (BAM output files from either TopHat or Bowtie) were first merged then assembled into transcripts using Cufflinks. Normalized FPKM (Fragments Per Kilobase of transcript per Million mapped reads) values were hierarchically clustered using Pearson's uncentered correlation coefficient with a centroid linkage. Clustered data were depicted graphically (heatmap and dendrogram) using JavaTreeView [25]. Significantly enriched GO terms were identified using the web based Gene Ontology Enrichment Analysis Software Toolkit [26] with the FDR set to 0.1 .

\section{Protein isolation and ms-based proteomic analysis}

D. immitis tissue samples were lysed in $1 \%$ SDS by sonication (3 times for $30 \mathrm{~s}$ ) followed by incubation at $65{ }^{\circ} \mathrm{C}$ for $30 \mathrm{~min}$ with gentle rocking. Samples $(100 \mu \mathrm{L}$ cell lysate) were then prepared for 2D-LC-MS/MS based proteome analysis using the filter aided sample preparation (FASP) kits (Expedion) with modifications to the protocol for simplification (for a complete protocol see [27]). Samples were digested on-filter with $10 \mu \mathrm{g}$ of Trypsinultra Mass Spectrometry Grade (New England Biolabs) overnight at $37^{\circ} \mathrm{C}$. Digested samples were eluted into a clean collection tube via three steps of centrifugation at $14,000 \times \mathrm{g}$ for $15 \mathrm{~min}$ as follows: Step 1: $80 \mu \mathrm{L} 50 \mathrm{mM}$ ammonium bicarbonate solution; Step 2: $50 \mu \mathrm{L} 0.5 \mathrm{M}$ sodium chloride solution (provided with the FASP kit); Step 3: $170 \mu \mathrm{L} \mathrm{H}_{2} \mathrm{O}$ with $0.1 \%$ formic acid. The final solution $(\sim 300 \mu \mathrm{L})$ was split into 2 aliquots per sample and frozen at $-20{ }^{\circ} \mathrm{C}$ until analysis by 2D-LC-MS/MS. The eluted solution was ready to load onto a $2 \mathrm{D}$-nano LC column without further purification.

The resultant complex peptide mixtures from the individual samples were loaded onto a biphasic C18-SCX (reverse phase-strong cation exchange) (Phenomonex)

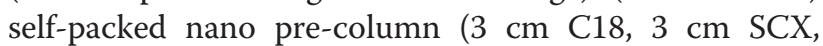
$150 \mu \mathrm{m}$ ID) that serves as the first dimension of the 2DLC system to capture peptides and wash away salts. Once loaded, the column was moved in-line with a U3000 HPLC (Dionex, subsidiary of Thermo Fisher Scientific) split to obtain $\sim 300 \mathrm{~nL} / \mathrm{min}$ flow rate over the nano-analytical columns. The pre-column was washed with $100 \%$ aqueous solvent followed by an organic solvent gradient ( $70 \%$ acetonitrile, $0.1 \%$ formic acid) to remove salts and move the peptides to the SCX phase. The pre-column was then attached to a $15 \mathrm{~cm} \times 100 \mu \mathrm{m}$ C18 front resolving column with an integrated nanospray tip (New Objective Picofrit) packed with Phenomenex Aqua C18. The resolving column was housed in a nanospray source (Proxeon, Thermo Fisher Scientific) attached to a QExactive mass spectrometer (Thermo Fisher Scientific). An automated $22 \mathrm{~h}$ two-dimensional LC-MS/MS run was programmed into Xcalibur (Thermo Fisher Scientific) and each sample was analyzed with a separation scheme consisting of eleven salt pulses followed by $2 \mathrm{~h} \mathrm{C18}$ separation (for more details on liquid chromatography method see [27]). During each analysis and all sample runs, the QExactive settings were as follows: the normalized collision energy for HCD was $28 \mathrm{eV}$, a full scan resolution of 70,000 K from 400- 
$1600 \mathrm{~m} / \mathrm{z}$, a HCD MS/MS resolution of 17,500 with an isolation width of $3 \mathrm{~m} / \mathrm{z}$, and the dynamic exclusion was set at $15 \mathrm{~s}$. Peptides were not excluded based on charge state and 1 microscan for both full and MS/MS scans were acquired. All MS and MS/MS data were acquired in profile mode.

\section{Proteome informatics}

All resultant MS/MS spectra from individual $24 \mathrm{~h}$ runs were searched with the Proteome Discoverer V. 1.4 (Thermo Fisher Scientific) and filtered via reverse database searching with maximum false positive rate of 0.5 . The Proteome Discoverer settings were as follows: HCD MS/MS, included a fixed modification for carboxyamidomethylated cysteines, a variable modification for urea carbamylation of arginine and lysine residues, fully tryptic peptides only, up to 4 missed cleavages, a precursor mass tolerance of $10 \mathrm{ppm}$ and a fragment mass tolerance of $0.6 \mathrm{Da}$. Only proteins identified with two fully tryptic peptides from a $22 \mathrm{~h}$ run were considered for further analysis. Tandem MS/MS spectra were searched against a combined protein database of $D$. immitis $(12,893$ entries) and $w \mathrm{Di}$ (871 entries).

As with the RNA-seq data, hierarchical clustering analysis was performed on the resultant filtered proteome datasets using Cluster 3.0 [25]. Peptide spectral count values were hierarchically clustered using Pearson's uncentered correlation coefficient with a centroid linkage. Clustered data were depicted graphically (heatmap and dendrogram) using JavaTreeView [25]. Significantly enriched GO terms were identified using the web based Gene Ontology Enrichment Analysis Software Toolkit [26] with the FDR set to 0.1 .

\section{Results and discussion}

\section{D. immitis tissue transcriptome overview}

Over 30 million single-end $50 \mathrm{bp}$ reads were generated using total RNA from various $D$. immitis tissues, $~ 84 \%$ of which mapped with high quality to the $D$. immitis reference genome (Table 1). The remaining $\sim 16 \%$ of reads are likely composed of Wolbachia transcripts and contaminants (dog and human). Of the mappable reads, approximately $87 \%$ were uniquely mapped to the $D$. immitis genome, while the remaining $\sim 12.3 \%$ of reads (including ribosomal RNA) mapped to multiple locations within the genome. Importantly, D. immitis gene model coverage and FPKM (Fragments Per Kilobase of transcript per Million mapped reads) distribution did not vary significantly among the different tissues (Additional file 1: Figure S1A). Close correlation was observed between biological replicates from the D. immitis tissues, except the uterus (Additional file 1: Figure S1B-F). Interestingly, the uterus displayed the highest transcriptional variability between biological replicates $\left(R^{2}=0.73\right)$, possibly due to the different infection ages of the worms in each sample (one sample was 1 year at harvest, the other sample was 4 years old at harvest).

Table 1 Overview of D. immitis tissues analyzed in current study

\begin{tabular}{|c|c|c|c|c|c|c|c|}
\hline \multirow{2}{*}{$\begin{array}{l}\text { Sample } \\
\text { FBW }\end{array}$} & \multirow{2}{*}{$\begin{array}{l}\begin{array}{l}\text { Total reads } \\
\text { (millions) }\end{array} \\
1.8\end{array}$} & \multirow{2}{*}{$\begin{array}{l}\text { High quality million } \\
\text { mapped reads } \\
1.5\end{array}$} & \multirow{2}{*}{$\begin{array}{l}\text { High quality million } \\
\text { mapped reads (combined) } \\
2.6\end{array}$} & \multirow{2}{*}{$\begin{array}{l}\text { Average coverage } \\
\text { (FPKM)/transcript }\end{array}$} & \multirow{2}{*}{$\begin{array}{l}\% \text { of all predicted } D \text {. immitis } \\
\text { transcripts detected } \\
55\end{array}$} & \multicolumn{2}{|c|}{$\begin{array}{l}\text { Number of D. immitis } \\
\text { proteins detected }\end{array}$} \\
\hline & & & & & & 1800 & 2408 \\
\hline & 1.3 & 1.1 & & & & 1912 & \\
\hline & & & & & & 1324 & \\
\hline \multirow[t]{2}{*}{ FU } & 1.9 & 1.6 & 3.8 & 66.8 & 74 & 3341 & \\
\hline & 2.5 & 2.2 & & & & & \\
\hline \multirow[t]{2}{*}{$\mathrm{FH}$} & 1.5 & 1.3 & 2.6 & 25.4 & 56 & 1100 & \\
\hline & 1.5 & 1.3 & & & & & \\
\hline \multirow[t]{2}{*}{$\mathrm{FI}$} & 1.8 & 1.5 & 3.3 & 38.2 & 60 & 202 & \\
\hline & 2.1 & 1.8 & & & & & \\
\hline \multirow[t]{2}{*}{ MBW } & 7.4 & 5.1 & 8.5 & 70.1 & 64 & - & \\
\hline & 5.1 & 3.4 & & & & & \\
\hline Ml & 7.6 & 4.5 & 4.5 & 58.7 & 69 & - & \\
\hline MT & 7.4 & 4.7 & 4.7 & 61.8 & 76 & - & \\
\hline MS & - & - & - & - & - & 35 & \\
\hline $\mathrm{MH}$ & - & - & - & - & - & 1183 & \\
\hline
\end{tabular}

D. immitis tissues (FBW female body wall, FU female uterus, FH female head, Flfemale intestine, MBW male body wall, $M I$ male intestine, $M T$ male testis, MS male spicule, $M H$ male head) analyzed by RNA-seq and/or MS-based proteomics. Values from each biological replicate (tissue samples from different worms) analyzed by each method are presented. Technical replicates were pooled prior to further analysis. Total number of unique proteins identified are presented for each tissue (bracketed for FBW biological replicates) 
Reads from the D. immitis tissues were also mapped to the Wolbachia endosymbiont ( $w \mathrm{Di}$ ) genome (Table 2 ). As anticipated, significantly fewer reads mapped to $w \mathrm{Di}$ genes (Table 2) than to $D$. immitis genes (Table 1) in each tissue: on average $\sim 9 \%$ of sequenced reads (ranging from 0.004 to $25 \%$ depending on the tissue) mapped to Wolbachia. A relatively high number of $w \mathrm{Di}$ transcripts $(\sim 90 \%)$ were detected by sequencing total RNA from $D$. immitis hypodermal tissues (female head, female body wall and male body wall), fewer transcripts were detected in the $D$. immitis uterus ( $70 \%$ of transcripts) and as expected very few Wolbachia transcripts were detected in the intestine and testis samples $(\leq 10 \%)$ (Table 2).

Inclusion of recently published whole-worm adult male (AM) and adult female (AF) transcriptomes [16] in the cluster analysis of the $D$. immitis tissue transcriptomes revealed the adult worm transcriptional profiles were most similar to their respective sex organs (testis or uterus) (Fig. 1a). Unlike the D. immitis AM and AF transcriptomes, which are dominated by the testis or uterus gene expression profiles, the AM and AF Wolbachia transcriptomes were most similar to the body wall and head tissue (which includes body wall) transcriptomes (Fig. 1b).

\section{Tissue-associated expression}

To identify groups of genes preferentially transcribed in specific tissues, hierarchical clustering was performed using the FPKM values of all $D$. immitis protein-coding genes expressed (Additional file 2: Dataset S1) in the seven tissues examined by RNA-seq. The resulting heatmap indicates
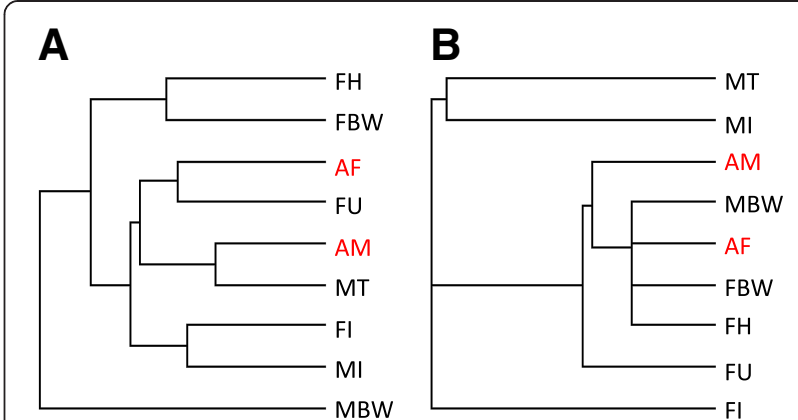

Fig. 1 Global comparison of D.immitis tissue transcriptomes. Hierarchical clustering reveals similarities between the transcriptional profiles of D. immitis (a) and wDi (b) from different tissues (FH female head, FBW female body wall, FU female uterus, MT male testis, Fl female intestine, MI male intestine, MBW male body wall) and whole adult worms (AF adult female, AM adult male, from [16], shown in red)

sets of genes expressed predominantly within a tissue or groups of tissues (MI/FI, FU/MT) (Fig. 2). These tissueassociated gene sets were then classified using GO terms which relate genes to biological function. GO enrichment analysis revealed that transcription of genes associated with carbohydrate (glycogen) processes and collagen formation (peptide-crosslinking) was functionally enriched in the FBW-associated gene set (Additional file 3: Table S1, Fig. 3). Although the FH and FBW samples display similar transcriptional patterns (Fig. 2), no functional enrichment was observed for the small subset of uniquely $\mathrm{FH}$-associated transcripts. Interestingly, neurotransmitter activity and carbon utilization (one-carbon metabolic processes and

Table 2 Wolbachia Transcriptome and Proteome Coverage in D. immitis Tissues

\begin{tabular}{|c|c|c|c|c|c|c|}
\hline Sample & wDi mapped reads & wDi mapped bases (millions) & $\begin{array}{l}\text { Average coverage } \\
\text { (FPKM)/transcript }\end{array}$ & $\begin{array}{l}\% \text { of all predicted } w D i \\
\text { transcripts detected }^{\mathrm{a}}\end{array}$ & $\begin{array}{l}\text { Number of } w D i \\
\text { proteins detected }\end{array}$ & \\
\hline \multirow[t]{3}{*}{ FBW } & 304,004 & 21.9 & 229.5 & 90 & 134 & 161 \\
\hline & 443,364 & 31.9 & & & 92 & \\
\hline & & & & & 59 & \\
\hline \multirow[t]{2}{*}{ FU } & 15,109 & 1.1 & 922.0 & 69 & 33 & \\
\hline & 82,897 & 6.0 & & & & \\
\hline \multirow[t]{2}{*}{$\mathrm{FH}$} & 308,412 & 22.2 & 216.3 & 86 & 34 & \\
\hline & 202,058 & 14.5 & & & & \\
\hline $\mathrm{Fl}$ & 1152 & 0.08 & 1627.3 & 7.1 & 0 & \\
\hline \multirow[t]{2}{*}{ MBW } & 440,851 & 22.0 & 621.5 & 97 & - & \\
\hline & 337,307 & 16.9 & & & & \\
\hline $\mathrm{Ml}$ & 1111 & 0.05 & 1536.2 & 10 & - & \\
\hline MT & 264 & 0.01 & 1367.9 & 8.3 & - & \\
\hline MS & - & - & - & - & 0 & \\
\hline $\mathrm{MH}$ & - & - & - & - & 62 & \\
\hline
\end{tabular}

a Based on number of transcripts expressed (FPKM >0) per life cycle stage. Values from each biological replicate (tissue samples from different worms) analyzed by each method are presented. Values from each biological replicate (tissue samples from different worms) analyzed by each method are presented. Technical replicates were pooled prior to further analysis. Total number of unique proteins identified are presented for each tissue (bracketed for FBW biological replicates) 


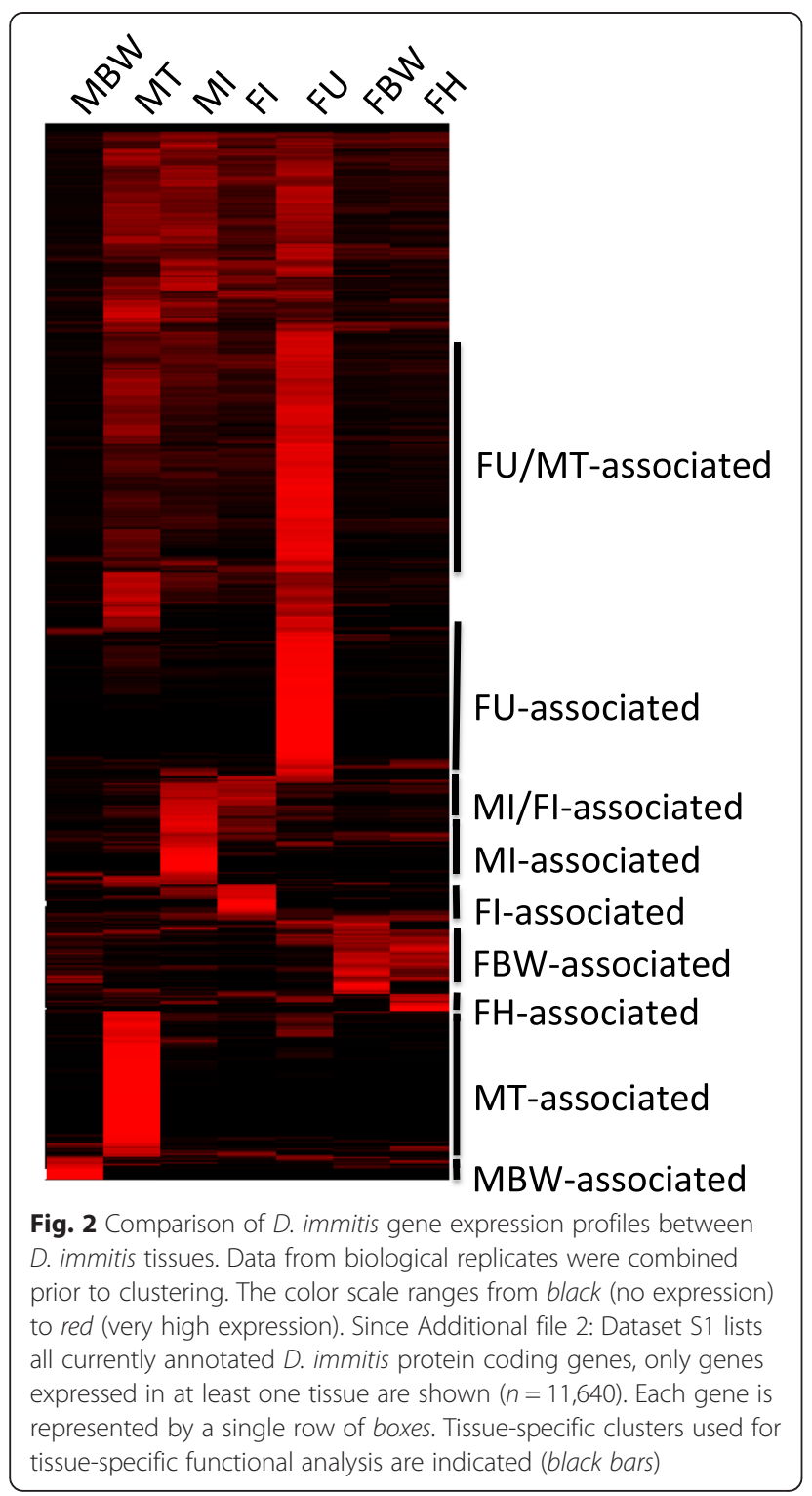

carbonate dehydratase activity) were enriched among transcripts associated with the MBW (Additional file 3: Table S1, Fig. 3). Perhaps unsurprisingly, functions such as transport and receptor activity were enriched among transcripts associated with the nematode intestine (MI/FI-associated), as were a number of functions seemingly unrelated to common intestinal functions (catecholamine metabolic processes, cytoskeletal/actin binding and turning behavior involved in mating) (Additional file 3: Table S1, Fig. 3). The largest gene cluster was expressed predominantly in the sex organs of both males and females (FU/MT-associated). Within this cluster, GO analysis revealed enrichment of genes involved in the cell cycle/mitosis, DNA repair/replication, RNA processing, gene expression and germ-line sex-determination (Additional file 3: Table S1, Fig. 3). Additionally, an unshared cluster of genes was also specifically associated with each sex organ (MT or FU). GO enrichment of these gene sets uncovered additional functions uniquely associated with the uterus (regulation of metabolic processes, DNA binding and protein methyltransferase activity) and testis (phosphorylation/dephosphorylation, trehalose metabolic processes, striated muscle contraction and pseudopodium) (Additional file 3: Table S1, Fig. 3). Many of the GO terms enriched within the $D$. immitis uterus and testis were previously identified as functions enriched in the ovary and testis of A. suum [17].

Many of the previously predicted enriched functions within adult worm transcriptomes [16] appear to arise from enriched functions in specific tissues, especially the testis. Indeed, 35 out of 74 GO terms previously identified as enriched within either $D$. immitis adult males or females were enriched within a specific tissue (Additional file 4: Table S2). Thirty GO terms enriched among male testis transcripts were also significantly enriched in the wholeworm AM transcripts [16], further suggesting the AM transcriptome is strongly influenced by the testis transcripts (Additional file 4: Table S2). Furthermore, the enrichment was typically more significant in the testis tissue than whole-worm adult male transcriptome (Additional file 5: Figure S2). Many of these GO terms (MT and AMassociated) were also enriched within the female uterus sample (Additional file 4: Table S2), however, out of the $15 \mathrm{GO}$ terms enriched in the D. immitis whole-worm adult female transcriptome none were shared with the $D$. immitis uterus transcriptome.

As expected, more $w$ Di transcripts were detected in nematode tissues known to contain Wolbachia (FBW, FH which includes body wall tissue, FU and MBW, Table 2). Nonetheless, transcripts of $w \mathrm{Di}$ genes were identified in every tissue transcriptome, including the testis and intestine samples. However, due to the especially low abundance of $w$ Di transcripts in the FI samples, mapped reads for the two FI biological replicates were merged prior to transcript assembly (Cufflinks) and differential expression testing (Cuffdiff). Interestingly, much lower Wolbachia transcript coverage was observed in the $D$. immitis female uterus than the female body wall (69 vs. $90 \%$, Table 2) and may be due to lower Wolbachia numbers per cell in the uterus tissue, high levels of nematode transcription (associated with rapid cell division) in uterus tissue (Table 1) or Wolbachia within the body wall tissue may be more transcriptionally active than Wolbachia within the uterus tissue.

Hierarchical clustering of Wolbachia transcriptomic data from $D$. immitis tissues also revealed clusters of Wolbachia genes preferentially transcribed in certain tissues (described in further detail below) (Additional file 6: Figure S3, Additional file 7: Table S3), however, no significant GO term enrichment was observed within any of these tissue-associated gene clusters. 


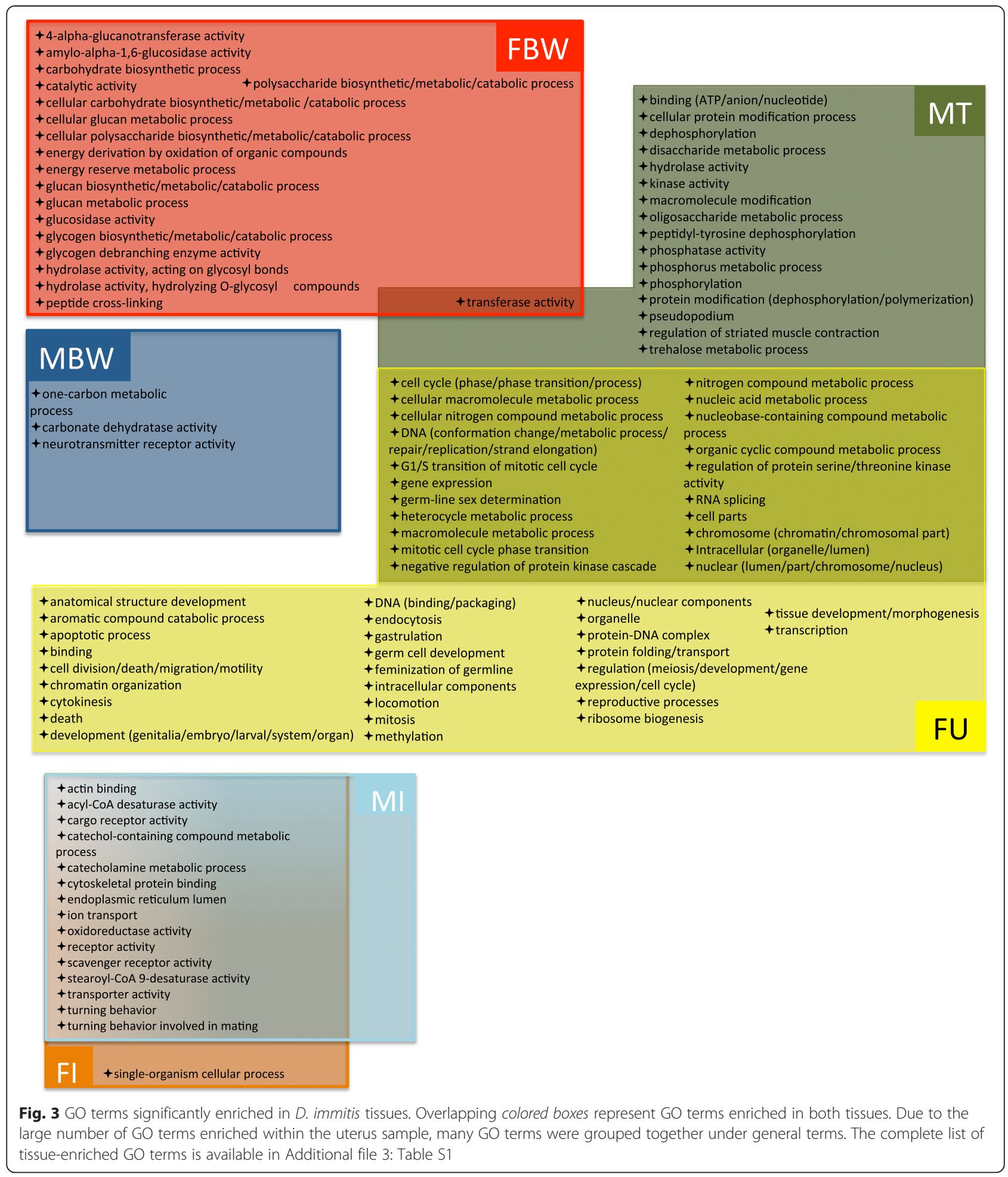

\section{Gender differences}

\section{D. immitis body wall}

Through its involvement in protein secretion as well as host immune evasion, the body wall and cuticle of a filarial nematode comprise the critical interface of the parasite-host interaction. Only 113 transcripts were differentially expressed between the male and female body wall (Additional file 8: Table S4). Approximately $50 \%(n=59)$ of these genes were upregulated in the MBW and include transcripts for major sperm proteins (potentially caused by contaminating transcripts from ruptured testis during dissection), cuticular collagens, an 
RNA helicase, a matrix metalloproteinase-like protein, a zinc finger-like protein and egl-9, a dioxygenase necessary for normal muscle function in C. elegans [28].

Transcripts for a number of proteins involved in muscle function, including myosin, actin binding proteins such as kettin or ketn-1, titin family member $t t n-1$, troponin $\mathrm{t}$, ph domain-containing protein 4, lim domain and actin-binding protein 1 and the alpha-actinin homologue $a t n-1$ were among the remaining 54 genes upregulated in FBW. Additionally, unc-89 and unc-22 (also known as twitchin), located in muscle A-bands of striated muscle and required for normal muscle function in C. elegans $[29,30]$, were upregulated in the D. immitis FBW. Other transcripts upregulated in the FBW compared to MBW include those for filarial antigen, a NAALADase ii peptidase protein, as well as LDL and HDL binding proteins. Additionally, the him-4 (high incidence of males) transcript that encodes a hemicentin was upregulated in the D. immitis FBW. Hemicentin is an evolutionarily conserved extracellular matrix protein secreted by skeletal muscle and the gonad that is involved in cell adhesion, migration and tissue attachment to the epidermis [31, 32].

\section{Wolbachia expression differences in the body wall of $D$. immitis}

Only 33 Wolbachia transcripts were differentially expressed between the female and male body wall samples (Additional file 9: Table S5). However, all of these transcripts were upregulated in the MBW. Among these transcripts were five hypothetical proteins, as well as $w$ Di genes involved in heme biosynthesis (hemB), purine biosynthesis (purS) and the type IV secretion system (T4SS) (VirB6). In agreement with the differential gene expression results (Additional file 9: Table S5), hierarchical clustering revealed MBW-associated $w \mathrm{Di}$ genes were involved in purine, heme and folate metabolism as well as secretion (Sec translocase) (Additional file 6: Figure S3, Additional file 7: Table S3). Although all genes in these biosynthetic and transport pathways were not upregulated in the male body wall, these results may suggest important biological differences in the Wolbachia symbiosis in males (which only contain Wolbachia in the lateral cords) and females (which contain Wolbachia in the ovaries and developing embryos as well as the lateral cords).

Although no Wolbachia genes were significantly upregulated in the $D$. immitis female body wall compared to the male body wall, hierarchical clustering of $w \mathrm{Di}$ expression data revealed four hypothetical proteins, one gene involved in isoprene biosynthesis and a gene involved in gluconeogenesis were present among the 9 FBW-associated Wolbachia genes (Additional file 6: Figure S3, Additional file 7: Table S3).

\section{D. immitis intestine}

More gender-associated differences were observed when comparing the $D$. immitis intestinal transcriptomes $(n=337)$ than the body wall transcriptomes $(n=113)$ (Additional file 8: Table S4). Among the FI upregulated transcripts were eat-18, $n h r-49$ and $n h r-31$. Originally identified as a feeding-defective mutation in C. elegans [33], eat-18 is required for nicotinic excitation of the pharyngeal $\mathrm{MC}$ neuron and thus, rapid pharyngeal pumping [34]. Of the two nuclear hormone receptors, $n h r-49$ appears to function as a key regulator of nutrient response and fat metabolism by influencing the expression of other metabolism related genes in C. elegans $[35,36]$. Transcription factor activity of the other nuclear hormone receptor upregulated in FI, $n h r-31$, is required for proper growth, development, and function of the excretory cell in C. elegans [37].

Notable among the transcripts upregulated in male intestine (compared to female intestine) were transcripts for a tubby protein homolog and lam-3. First identified in mice [38], when mutated, tubby proteins cause delayed onset obesity. Later studies in C. elegans confirmed the role of tubby proteins in fat and carbohydrate metabolism [39]. Required for normal tissue development (including pharyngeal development), laminins, including lam-3 are expressed in the pharynx and hypodermis, and also weakly in the intestine of $C$. elegans [40]. Additionally, a number of potential transcription factors including zinc finger proteins, kruppel like factor, ef hand containing proteins and calmodulin-dependent calcium sensors were upregulated in the male compared to female intestine.

\section{Wolbachia expression in D. immitis intestine}

Not surprisingly, very few Wolbachia genes were transcribed in the intestine of either $D$. immitis sex $(n=62$ transcripts in the female intestine, $n=91$ in the male intestine) (Table 2). Interestingly, however, of those Wolbachia transcripts detected in the intestine samples, expression levels (FPKM values) were relatively high compared to the other tissues tested (Additional file 6: Figure S3).

Only $15 w$ Di transcripts were expressed in both intestinal tissues, including four hypothetical proteins. Interestingly, in support of previous Wolbachia transcriptomic data from the cattle parasite Onchocerca ochengi that suggests Wolbachia may provide energy (ATP) to its host in certain tissues [41], transcripts for a number of $w \mathrm{Di}$ proteins/enzymes involved in energy production (including cytochrome $C$, ATP synthase $\beta$ chain and isocitrate dehydrogenase) were detected in both male and female $D$. immitis intestine. Hierarchical clustering of the Wolbachia gene expression data from the $D$. immitis tissues (Additional file 6: Figure S3) revealed a number of 
$w$ Di proteases among the 42 genes preferentially transcribed in the FI, as well as a phosphate transporter and a heme exporter $(\mathrm{CcmB})$ (Additional file 7: Table S3). Prominent among the MI-associated Wolbachia transcripts were genes involved in secretion (T4SS, Sec and Tat translocase components), as well as genes involved in isoprene, riboflavin and peptidoglycan biosynthesis (Additional file 7: Table S3). Exactly how these transcripts were detected in a tissue shown to be completely devoid of Wolbachia [5] remains unclear; however, it is possible these Wolbachia transcripts were exported from other nematode tissues. Notably, no Wolbachia peptides were detected by mass spectrometry in the $D$. immitis female intestine (see below).

\section{D. immitis reproductive tissues}

The reproductive tissues, i.e., testis and uterus, are a significant source of excretory-secretory products of filarial nematodes [42], known to elicit a significant immune response in filarial nematode infected animals: serum from Acanthocheilonema viteae-infected rodents strongly reacts with both male and female reproductive tissues [43]. Previous cluster analysis of four nematode proteomes (B. malayi, C. elegans, Trichinella spiralis and A. suum) with the predicted $D$. immitis nuclear proteome (version 1.3) identified 850 conserved proteins uniquely shared by $D$. immitis and $B$. malayi, but lacking in the three other nematode species [14]. Previous BLASTP analysis with the improved version $2.2 \mathrm{D}$. immitis proteome removed redundancies and yielded 834 predicted proteins which may represent common filarial nematode targets shared between D. immitis and B. malayi [16]. Assessment of tissue-specific transcription of these 834 gene products is listed in Additional file 8: Table S4. Many of these 834 genes $(n=523)$ displayed tissueassociated transcriptional patterns. Of these $523 \mathrm{D}$. immitis genes with tissue-associated transcriptional patterns, over $80 \%(n=421)$ were associated with a reproductive tissue (FU, MT or FU/MT) of $D$. immitis. Over 200 of these transcripts expressed in the FU were confirmed by proteomics (see below). It is clear the reproductive tissues express transcripts and secrete proteins that may serve to target filarial nematode reproduction in a transmission blocking approach to disease control. Perhaps unsurprisingly, the pairwise comparison of the uterus and testis sample produced the largest number ( $n=2998$, Additional file 9: Table S5) of differentially expressed genes compared to any other tissue comparison by gender (FBW v. MBW or FI v. MI).

Genes upregulated in the $D$. immitis uterus Of the nearly 3000 transcripts differentially expressed between the D. immitis uterus and testis tissues, 2300 are upregulated in the uterus. A number of notable transcripts were expressed in the uterus tissue, but not expressed at all in the male testis. These include the follicle stimulating hormone receptor, $n h r-23$, unc-7, unc-9, $u n c-62$, odr-3, a zona pellucida-like domain containing protein, GABA and dopamine transporters, riboflavin kinase and phosphomevalonate kinase. A member of the nuclear hormone receptor family of transcription factors [44], nhr-23 (through the regulation of collagen and hedgehog related genes [45]) has been shown to be critical to all four larval molts in C. elegans [46] and may therefore represent a transcript that is highly expressed in the intrauterine microfilariae of $D$. immitis in preparation for future molts. The three uncoordinated (unc) proteins expressed in the uterus but not expressed in the testis (unc-7, -9 and -62$)$ are all implicated in female reproductive development in C. elegans: unc-7 and unc-9, both gap junction forming innexins, are required for normal egg laying $[47,48]$ and $u n c-62$ is necessary not only for vulval morphogenesis but also posterior embryonic morphogenesis [49]. Curiously, another gene expressed in the $D$. immitis uterus tissue but not in the testis was $o d r-3$, which encodes for a G-protein subunit typically expressed in neurons and required for normal chemotaxis in C. elegans [50].

Among transcripts expressed in both the uterus and testis, but upregulated in the female reproductive tract were transcripts for doublesex, mab-3-related transcription factor 1, transcription factor bigmax, apoptosis regulatory protein, placental protein $11, \mathrm{DNA}$ damagebinding protein 1 , chitin synthase 1 (chs-1), cannabinoid receptor 1, gld-2, nab-1, unc-112 related protein and a number of nuclear hormone receptors $(n h r-6, n h r-7 a$, $n h r-14, n h r-41$ and $n h r-91)$. Of these nuclear hormone receptors, $n h r-6$, an orthologue of the Drosophila melanogaster E75 gene which plays a role in molting, was previously shown to be highly expressed in $D$. immitis adult females compared to adult males [51], while nhr14 controls gene expression in response to estrogen in C. elegans [52]. Other transcripts upregulated in the $D$. immitis uterus have been implicated in germ line (let-49 [53], sax-7 [54], sel-12 [55]), oocyte (mep-1 [56], daz-1 [57]) or embryonic (chitin synthase 1 [58], nrf-6 [59], die-1 [60], gei-17 [61]) development in C. elegans. Interestingly, two of these genes (chitin synthase I and nrf-6) upregulated in the uterus compared to the testis were predicted to be among the top 40 drug targets for the related filarial nematode, B. malayi [62].

Genes upregulated in the D. immitis testis A total of 698 transcripts were upregulated in the male testis compared to the female uterus, nearly $80 \%$ of which $(n=471)$ displayed no expression in the uterus (Additional file 9: Table S5). Perhaps not surprisingly, the list of genes 
upregulated in the testis (which contains sperm) compared to the uterus includes a number of major sperm proteins. Also upregulated in the D. immitis testis were transcripts for fog-3, gld-1, spe-4 and spe-9. In C. elegans, fog-3 is most highly expressed in the L3, L4 and early adult stages and is essential for spermatogenesis [63]. Additionally, gld-2, along with gld-3, activates gld-1 to regulate entry into meiosis during germ cell development [64]. In the D. immitis tissues, gld-2 was expressed in both testis and uterus, but was upregulated in uterus, whereas gld-1 was only transcribed in the testis. Interestingly, in the absence of gld-1, gld-2 is still able to promote meiosis [64], suggesting that $g l d-2$ likely has other unidentified targets (particularly in the female germline where gld-1 is not transcribed but meiosis is occurring). Both spe- 4 and spe9, which were upregulated in the testis, are critical to spermatogenesis in C. elegans [65]. While spe-4 is involved in proper partitioning of cytoplasmic components during spermatogenesis [66], spe-9 mediates oocyte fertilization likely through an adhesive function or signaling event [67]. Also prominent among transcripts upregulated in the testis compared to the uterus were msp-domain containing proteins, a designated sperm-specific sodium proton exchanger, phosphatases and kinases, as well as and a number of hypothetical proteins conserved between $D$. immitis and related filarial nematodes B. malayi and L. loa.

\section{Wolbachia expression in D. immitis uterus and testis}

Among 86 Wolbachia genes preferentially transcribed in the D. immitis uterus (Additional file 7: Table S3) were genes involved in purine and pyrimidine biosynthesis, heme biosynthesis, isoprene biosynthesis, energy production, fatty acid biosynthesis and secretion (components of the Sec translocation system). Since Wolbachia are absent in the male germ line of filarial nematodes [5], very few Wolbachia transcripts were detected in the testis tissue of $D$. immitis (Table 2). Interestingly however, transcripts suggested to be involved in the symbiosis between filarial nematodes and Wolbachia were among the $w \mathrm{Di}$ MT-associated transcripts including those associated with secretion systems (components of the T4SS, the type 1 secretion system and the Sec translocase), purine and peptidoglycan biosynthesis as well as heme metabolism (Additional file 7: Table S3).

\section{D. immitis female head tissue expression}

The female head tissue included cuticle, body wall, mouth, amphidial neurons, nerve ring with some peripheral nerves as well as the pharyngeal muscle. Although no GO terms were enriched among the $\mathrm{FH}$-associated transcripts (Fig. 2), nearly 300 transcripts were upregulated in the $D$. immitis female head tissue when compared to other female tissues examined (FBW, FI or FU,
Additional file 9: Table S5). These head upregulated transcripts include those potentially linked to neuronal activity such as ptc-3 [68] and slo-2 [69, 70]. Muscleassociated transcripts for dim-1, unc-87 and unc-89 (Additional file 9: Table S5) were upregulated in $\mathrm{FH}$ compared to FI or FU, but were much higher in the FBW tissue than the head tissue, suggesting these highly expressed FH transcripts likely originate from the body wall muscle and not the pharyngeal muscle.

\section{Wolbachia expression in D. immitis female head}

Previous immunofluorescence staining confirmed the absence of Wolbachia in nerve ring of adult B. malayi [5]. Therefore it is not surprising that the Wolbachia transcriptome of the $D$. immitis female head closely resembles the Wolbachia transcriptome of the female body wall tissue (Additional file 6: Figure S3), since transcripts detected in the FH likely originate from the hypodermal cords in the cephalic region.

\section{Tissue comparisons}

\section{D. immitis body wall versus intestine}

Vaccination of laboratory animals with intestinal antigens from parasitic nematodes has conferred partial immunity to subsequent parasite infection [71-73], making intestinal antigens attractive vaccine targets. A thorough comparison of the D. immitis body wall and intestine transcriptomes may identify potential so-called 'hidden antigens' present in the intestine. Interestingly, fewer differences in gene expression were observed when comparing the female body wall and female intestine (387 differentially expressed genes) than the male body wall and male intestine (1110 differentially expressed genes) (Additional file 9: Table S5).

\section{Genes Upregulated in the D. immitis Female Intestine}

Of the genes differentially expressed between the $D$. immitis FBW and FI samples, nearly $70 \%(n=264)$ were upregulated in the intestine and could potentially represent 'hidden antigens' (Additional file 9: Table S5). Among these transcripts are gei-4, jun transcription factor homolog family member (jun-1), daf-16, hlh-30, pha-4 and unc-83. Concordant with their expression in the intestine, it was recently discovered in C. elegans that transcription factors hlh-30, jun-1 and daf-16 play a role in mediating fasting-induced longevity by inducing transcriptional changes and regulating proteolysis [74, 75]. Another transcription factor expressed in the female intestine but not body wall, pha-4, is required for pharynx development in C. elegans [76]. Although interesting, these intestine-associated transcription factors are likely unsuitable vaccine targets since they are probably not present on the surface of the intestine. 
Other transcripts upregulated in the female intestine compared to the female body wall include: fat-3, $m d t-15$, cyn-5, lap-1 and mrp-5. In C. elegans, both fat-3 and $m d t-15$ are critical to fatty acid metabolism: $m d t-15$ coordinates a number of metabolic factors that regulate the expression of fatty acid desaturase genes, such as fat-3 [77, 78]. A type B cyclophilin encoded by cyn-5, a leucine aminopeptidase encoded by lap-1 and the multi-drug resistance protein encoded by $m r p-5$ have all been shown to be expressed in the intestine of $C$. elegans [79-81]. Interestingly, detailed analysis in $C$. elegans revealed that mrp-5 serves as a critical heme transporter that exports intestinally absorbed heme to other tissues [81].

Genes Upregulated in the D. immitis Male Intestine Of the genes differentially expressed between the $D$. immitis MBW and MI, $55 \%(n=613)$ were upregulated in the intestine (Additional file 9: Table S5). Notable among these transcripts are gei-4, pha-4, fat-3, $m d t-15$ and $m r p-5$ (also upregulated in FI compared to FBW). Other transcripts upregulated in the D. immitis male intestine compared to the male body wall include $\exp -2$, nas-4 and itr-1. The exp-2 gene is required for normal pharyngeal pumping in C. elegans by encoding for a potassium channel that allows the pharynx muscles to repolarize quickly [82]. Expressed in the pharynx and intestine of C. elegans [83], nas-4 is a zinc metalloproteinase that likely plays a role in nematode digestion. The inositol 1,4,5-triphosphate receptor encoded by itr-1 is highly expressed in both the pharynx and intestine of C. elegans [84] and appears to play a role in pharyngeal and intestinal pumping [85].

\section{D. immitis body wall versus reproductive organs}

A similar number of differences in gene expression are observed when comparing the $D$. immitis female body wall to uterus tissue (1555 differentially expressed genes) and the D. immitis male body wall to testis tissue (1642 differentially expressed genes).

Of the differentially expressed genes between the FBW and FU, only $17 \%(n=268)$ are upregulated in the body wall (Additional file 9: Table S5). These transcripts include a number of muscle proteins (actin/myosin), collagens, filarial antigen, galectins and lectin binding proteins as well as transcripts for him-4, alp-1, unc-22, unc-87, unc-89, deb-1, ketn-1 and ttn-1. Importantly, alp-1, unc-87, deb-1, ketn-1 and ttn-1 have all been associated with the body wall muscle structure and/or function in C. elegans [86-92].

The remaining $83 \%(n=1287)$ of transcripts differentially expressed between the $D$. immitis FBW and FU are upregulated in the uterus tissue (Additional file 9: Table S5). Not surprisingly, many of these transcripts (die-1, gei-17, let-49, zona pellucida-like protein, gld-2, sax-7, nhr-7a, nhr-23, unc-7, unc-9 and unc-62) were also upregulated in the female uterus compared to the male testis. Interestingly, gei-4 and the transcription factor jun-1, which were both upregulated in the female intestine when compared to FBW (see above), are upregulated in the female uterus as well (when compared to the female body wall). In addition to its role in mediating fastinginduced transcriptional changes in the intestine, jun-1 also regulates phospholipase $\mathrm{C}$ expression, thereby affecting inositol triphosphate levels which in turn affect ovulation [93]. Other noteworthy transcripts upregulated in the uterus compared to the female body wall were histones, apr-1, the lipocalin encoding genes $l p r-4, l p r-5$ and $l p r-6$, a wnt family protein, the nuclear transport factor $n t f-2$ and the mucin-like protein encoded by let-653.

Nearly $40 \% \quad(n=656)$ of the genes differentially expressed between the D. immitis MBW and MT were upregulated in the body wall (Additional file 9: Table S5). As with the female body wall, these transcripts include muscle proteins such as actin, myosin, collagens as well as filarial antigen and galectins. Additionally, transcripts for unc-87, unc-95, mpz-1 and ttr-5 are upregulated in the male body wall compared to the testis. As mentioned above, unc-87 is critical for muscle development $[87,88]$. The muscle LIM domain containing protein encoded by unc-95 is required for locomotion and organization of thick and thin filaments in body wall muscle [94]. Interestingly, the $t$ tr- 5 gene encodes a transthyretin-like protein, which was among the most abundant proteins detected in a recent analysis of the excretory-secretory products of another filarial nematode, Litomosoides sigmodontis [42].

Many of the transcripts upregulated in the D. immitis MT compared to the MBW, including gei-4, pha-4, gld1, unc-7 and unc-9 have been described in detail above. Additionally, transcripts for a posterior sex combs protein, trehalase, msp domain containing proteins, a dad (defender against death) family protein and ssp-35 (sperm specific family protein, class $\mathrm{P}$ ) were upregulated in the testis compared to the male body wall.

\section{Comparison of Wolbachia expression in D. immitis female body wall and uterus}

A thorough comparison of Wolbachia expression profiles within the germ line and the somatic cells (hypodermis of the body wall) may provide clues as to the varying role of Wolbachia within the different nematode tissues inhabited by the endosymbiont. A total of 121 Wolbachia transcripts were differentially expressed between the FBW and FU tissues, a large proportion of which ( 91 \%) were upregulated in the uterus (Additional file 10: Table S6) despite the fact that lower Wolbachia transcript coverage was 
observed in the D. immitis female uterus (69\%) than the female body wall sample (90\%) (Table 2). Only $11 \mathrm{wDi}$ transcripts were upregulated in the female body wall. These include transcripts for a component of the Sec translocase, components of the T4SS as well as the heme $($ hem $C$ ) and pyrimidine biosynthesis pathways (Additional file 10: Table S6), all of which have been previously implicated in the symbiotic relationship between filarial nematodes and Wolbachia. Notable among the Wolbachia transcripts upregulated in the $D$. immitis uterus include transcripts for a number of other steps in the heme biosynthetic pathway (hemB, hemD, hemE), the T4SS (VirB9 and VirB11) and the Sec translocase ( $\operatorname{SecB}$ and $f f h$ ) (Additional file 10: Table S6). Additionally, RibA, the first gene in the pathway leading to riboflavin synthesis, a putative Wolbachia-nematode shared metabolite, was another significantly upregulated Wolbachia transcript in the D. immitis uterus compared to the female body wall.

Our comparison of the $w \mathrm{Di}$ transcriptomes from the D. immitis uterus and female body wall strongly echoes previous findings that found transcripts involved in translation and DNA replication were upregulated in the germ line Wolbachia transcriptome from O. ochengi $(w \mathrm{Oo})$ in comparison to that of the somatic transcriptome [41]. Notably in terms of translation, elongation factor Tu, 3 ribosomal proteins and polypeptide deformylase were all upregulated in $w$ Oo germ line transcriptome compared to the somatic transcriptome. Likewise, we find $w \mathrm{Di}$ transcripts for elongation factor Ts, 7 ribosomal proteins, a peptide deformylase and a number of tRNA synthetases are all upregulated in D. immitis uterus tissue compared to the female body wall tissue (Additional file 10: Table S6). Additionally, Wolbachia transcripts related to DNA replication and repair including a uracil DNA glycosylase and DNA recombination proteins RmuC and MutL were upregulated in the $D$. immitis germ line tissue (uterus) compared to the somatic tissue (female body wall). As previously suggested [41], these Wolbachia transcripts associated with transcription and DNA replication that are upregulated in the germ line tissue may be related to the fact that prior to fertilization, Wolbachia appears to divide rapidly in the germ line of female filarial nematodes [5]. Interestingly, two Wolbachia endonuclease transcripts, endonuclease III and Nuc endonuclease, are also upregulated in the D. immitis uterus tissue. Since most filarial nematodes are unable to synthesize purines and pyrimidines de novo, it is possible that Wolbachia endonucleases are recycling nucleotides in order to supplement the host nucleotide pool within the rapidly dividing nematode germ line. Alternatively, Wolbachia may be utilizing the nucleotides for their own propagation within the germ line.

\section{D. immitis tissues proteome Overview}

Overall, 4162 of the 12,857 annotated proteins or 32\% of the predicted $D$. immitis proteome were verified by at least two unique peptides from the analysis of six $D$. immitis tissues (FBW: 2408; FU: 3341; FH: 1100; FI: 202; MH: 1183 and MS: 35 proteins) (Table 1, Additional file 11: Dataset S2). Higher protein coverage was obtained with B. malayi [95], however that level of coverage was attained using five life cycle stages whereas our data only covers the proteome of adult D. immitis tissues. A complete list of the peptide spectrum matching counts for every $D$. immitis protein detected, along with their corresponding gene annotation is provided in Additional file 12: Table S7. Likely due to incomplete lysis of the spicule due to its inherent structural stability, only 35 proteins were detected in this tissue (Additional file 12: Table S7). Because these proteins were also detected in another $D$. immitis tissue, the spicule sample was omitted from further analysis. Only $3.7 \%$ of identified proteins $(n=152)$ were common among the remaining five tissue samples (FBW, FU, FH, FI and MH) (Fig. 4a). Not surprisingly, these shared proteins include common structural and motor proteins (actin/collagens/kinesin), galectins, annexins, redox proteins (thioredoxin peroxidases), heat shock proteins, enzymes (enolase, ATP synthase components, lactate dehydrogenase, pyruvate kinase, protein disulfide isomerase, protein phosphatase) and clathrin, among others (Additional file 12: Table S7). Among the 4162 proteins identified, $49 \%(n=2056)$ were tissue specific (identified only in one tissue) (Fig. 4a, Additional file 12: Table S7). Perhaps unsurprisingly, the highest number of tissue specific proteins (1458) were identified in the uterus sample (Fig. 4a). Out of the 202 proteins identified in the $D$. immitis female intestine sample, only 6 proteins were intestine-specific. These potential hidden antigens include: aspartic protease sp-1, transmembrane protein 132a, guanine nucleotidebinding protein $\alpha-7$ subunit gpa-7, cell division protein kinase 5, $\alpha$-ulin (catenin vinculin related) family member ctn-1 and a hypothetical protein. Interestingly, both gpa7 and ctn- 1 are expressed in the pharyngeal muscle and intestinal muscle of $C$. elegans [96, 97].

Of the predicted 834 filarial-specific proteins (those uniquely shared by $D$. immitis and B. malayi, but lacking in C. elegans, T. spiralis and A. suum, Additional file 8: Table S4) [14], 285 were confirmed by proteomics of $D$. immitis tissues. The vast majority of these proteins were detected in the FU sample $(n=215)$, while $136,67,66$, 11 and 3 of these proteins were detected in the FBW, $\mathrm{FH}, \mathrm{MH}, \mathrm{FI}$ and $\mathrm{MS}$, respectively.

Filarial nematode genomes and corresponding predicted proteomes are riddled with hypothetical and unannotated proteins. Previous proteomic analysis of $B$. 

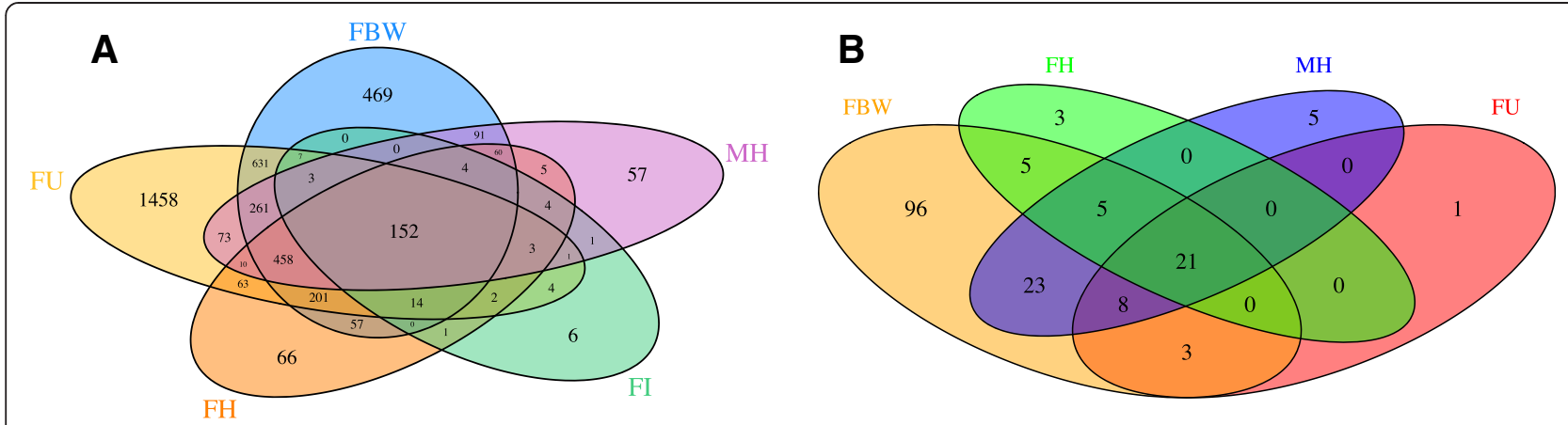

Fig. 4 Venn diagrams illustrating D. immitis (a) and wDi (b) proteins detected in D. immitis tissues. a Since only 35 D. immitis proteins were identified in the MS tissue sample, the spicule sample was excluded from this figure. However, those 35 proteins were also identified in other tissues and are represented accordingly. b Only FBW, FH, MH and FU samples are shown since no wDi proteins were detected in the FI or MS sample

malayi life cycle stages was able to confirm the existence of $\sim 2300$ hypothetical proteins [95]. Out of the $\sim 5000$ hypothetical and unannotated proteins within the $D$. immitis genome, our tissue-specific proteome has validated 744 'hypothetical' proteins (52 annotated as hypothetical proteins and 692 unannotated proteins) (Additional file 12: Table S7). The majority of these hypothetical proteins were detected in the FBW and FU tissues. Intriguingly, 128 of the 744 hypothetical proteins identified herein are putatively filarial-specific antigens (conserved proteins shared by D. immitis and B. malayi, but lacking in C. elegans, T. spiralis and A. suum, Additional file 8: Table S4) [14].

Hierarchical clustering of $D$. immitis proteins detected in the $6 \mathrm{D}$. immitis tissue samples revealed groups of proteins associated with specific tissues (Additional file 13: Figure S4). GO enrichment analysis of these clusters revealed significantly enriched functions within the FBW, FU, FI and $\mathrm{MH}$-associated protein clusters (Additional file 3: Table S1). Out of the 78 GO terms identified as enriched within the uterus sample by RNA-seq, 48 of those were also identified as enriched by $\mathrm{GO}$ analysis of the $\mathrm{FU}$-associated protein cluster, including those involved in cell cycle/mitosis/DNA replication, transcription, metabolic processes, cell parts (chromosome/organelle). In total, over 450 additional uterus-associated GO terms were identified by proteomic analysis. Notable among uterus-associated proteomic GO terms were those involved in anatomical structure development, apoptosis, reproductive processes, chromosome/chromatin organization, cytokinesis, embryo development, protein localization/targeting, gamete generation, gene silencing, mRNA processing and helicase activity.

\section{Wolbachia proteome in D. immitis tissues}

Proteomic analysis of the $D$. immitis tissues identified $170 w$ Di proteins $(\sim 20 \%$ of the 871 predicted protein encoding genes) based on at least two unique peptides matching a Wolbachia protein (Additional file 14: Table S8). A complete list of the peptide spectrum matching counts for every Wolbachia protein detected is provided in Additional file 14: Table S8. Likely due to the presence of biological replicates, the vast majority of Wolbachia proteins were detected in the FBW sample (Fig. 4b), while surprisingly very few were detected in the uterus tissue. Likely due to the absence of Wolbachia in these tissues, no $w$ Di proteins were detected in the FI and MS samples (Table 2). Similar to previous proteomics studies on $w \mathrm{Bm}[95]$ and $w \mathrm{Oo}$ [41], the most abundant Wolbachia proteins detected were chaperonins and heat shock proteins such as GroEL, DnaK, ClpB protein, GroES and surface antigen Wsp (Additional file 14: Table S8).

A number of biosynthetic pathways have been implicated in potential metabolite provisioning between $\mathrm{Wol}$ bachia and filarial nematodes [11] and may serve as possible Wolbachia-specific targetable pathways [16]. Components of many of these pathways, including the heme biosynthesis pathway ( 3 of 8 enzymes), the purine biosynthesis pathway ( 6 of 10 enzymes), and the isoprene biosynthesis pathway ( 1 of 5 enzymes) were detected, mainly in the D. immitis female body wall and head tissues (Additional file 14: Table S8). Likely due to the low coverage of the $w$ Di proteome, no peptides matching enzymes from Wolbachia pathways for the synthesis of pyrimidines (6 enzymes), lipid II/peptidoglycan (9 enzymes), riboflavin (5 enzymes) or folate (5 enzymes) were detected in any $D$. immitis tissue.

Annotation of the $w$ Di genome describes 277 of the 871 predicted proteins as hypothetical proteins. Based on our transcriptomic data, 264 of these hypothetical proteins were transcribed (FPKM values $>0$ ) in some $D$. immitis tissue (Additional file 10: Table S6). In total, 9 of the 264 transcribed hypothetical proteins were validated as genuine $w$ Di proteins by proteomic analysis (Additional file 12: Table S7). Interestingly, some of these 'hypothetical' proteins (namely wDi:fig_82301.12.peg.651, 
wDi:fig_82301.12.peg.439 and wDi:fig_82301.12.peg.677) were also among the 10 most abundant Wolbachia proteins detected (Additional file 14: Table S8).

\section{Comparison of transcriptomics and proteomics}

Often very little correlation exists between transcript and protein abundance [41], because RNA levels may not accurately reflect protein levels since protein levels in vivo are not only dictated by the rate of transcription/ translation, but also by the rate of protein degradation. In the $4 \mathrm{D}$. immitis tissues examined by both methods, 11,640 D. immitis transcripts and $4162 \mathrm{D}$. immitis proteins were detected. Overall, proteomic analysis of these adult $D$. immitis tissues revealed peptide evidence for nearly $36 \%$ (4149) of transcripts identified in tissues examined by both methods (Fig. 5). Based on individual tissue transcriptomics/proteomics, the female uterus sample had the highest percentage of $D$. immitis transcripts verified within that tissue by mass spectrometry ( $35 \%$ ), followed by the FBW, FH and FI $(32,14$ and $3 \%$, respectively) (Additional file 15: Figure S5A-D). Conversely, only $0.3 \%(n=13)$ of proteins identified lacked any transcriptional evidence in any $D$. immitis tissue examined (Fig. 5), the majority $(n=10)$ of which were detected in the female uterus. Only $\sim 2-7 \%$ of $D$. immitis proteins identified in a tissue lacked transcripts within that same tissue (Additional file 15: Figure S5A-D). These proteins may be transcribed in another tissue then trafficked to the tissues where they were detected by proteomic analysis. Interestingly, 9 proteins identified in the FBW sample lacked detectable transcription in any female tissue, but were transcribed in a male tissue(s).

All 170 Wolbachia proteins identified by at least 2 unique peptides in proteomic analysis were transcribed in at least one $D$. immitis tissue. The highest level of $w D i$ proteomic coverage of the transcriptome was observed in the D. immitis FBW (over $20 \%$ coverage), whereas only 6 and $5 \%$ of transcribed genes were verified in the $\mathrm{FU}$ and $\mathrm{FH}$ tissues, respectively (Additional file 16: Figure S6A-C). A total of 9 Wolbachia proteins were identified by proteomic analysis in a specific tissue

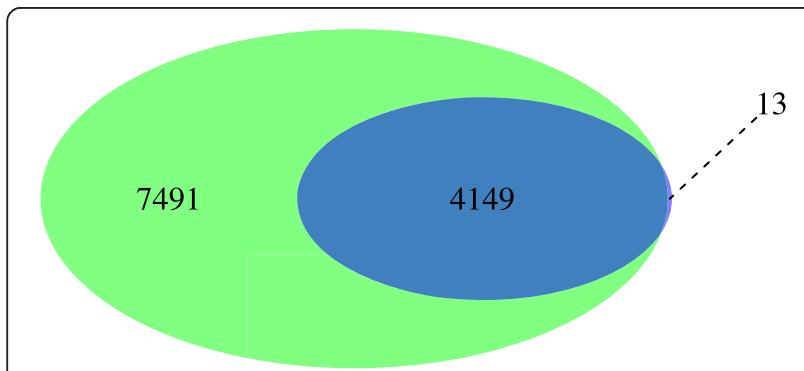

Fig. 5 Euler diagram illustrating $D$. immitis genes detected by transcriptomic (green) and proteomic (blue) methods in D. immitis tissues that was not confirmed by any transcriptomic evidence in that same tissue (Additional file 16: Figure S6A-C). It is possible these proteins were made either in another $D$. immitis tissue containing Wolbachia and then transported/secreted, or alternatively these proteins may have been transcribed during an earlier developmental stage of $D$. immitis prior to localization in tissues seemingly devoid of Wolbachia. How these proteins came to be in these tissues while their transcriptional levels were undetectable by RNA-seq remains unclear, but suggests Wolbachia proteins and their associated functions may extend beyond the tissue-specific localization of Wolbachia within the adult nematode.

\section{Conclusions}

More knowledge is gained with every filarial nematode genome sequence that is analyzed. While the advent of RNA-seq and more sensitive proteomic methods has also been of great value, these methods provide the most significant level of detail when used in concert with one another. Understanding the biology of filarial nematodes and the development of novel therapeutic strategies against them requires a greater spatial understanding of both nematode and Wolbachia gene expression and protein abundance. We therefore implemented parallel transcriptomic and proteomic analysis to study filarial nematode tissue-specific transcriptional patterns in order to gain functional information. Such spatial resolution of gene expression can provide greater understanding not only on how that specific tissue may interact with the vertebrate host (or evade the host immune system), but also how Wolbachia may differentially contribute to various nematode tissues. Notably, our analysis of $D$. immitis tissues revealed that the reproductive tissue (uterus and testis) transcriptomes most closely correlate with recently published whole-worm transcriptomes, while the Wolbachia transcriptome of the body wall tissue most closely correlated with the whole-worm Wolbachia transcriptomes. Together, transcriptomics and proteomics of D. immitis intestinal tissues highlighted a number of potential hidden antigens. Interestingly, some Wolbachia genes upregulated in nematode tissues include transport systems and genes predicted to be involved in the symbiotic relationship with $D$. immitis. Furthermore, the presence of Wolbachia transcripts and proteins in D. immitis tissues lacking Wolbachia suggests that the symbiont transcripts and proteins may traffic to other nematode tissues.

Not only has this study provided a new level of detail to our knowledge of filarial tissue transcription/translation, but also furthered our understanding of how $\mathrm{Wol}$ bachia endosymbionts may interact with filarial nematodes. For the first time in any filarial nematode, this data in conjunction with the recently published life 
cycle transcriptome [16], serves as a comprehensive atlas of the temporal and spatial representation of expression of both $D$. immitis and $w$ Di genes, including those that may be used to develop future therapeutic strategies.

\section{Availability of supporting data}

The mapped reads (TopHat BAM output files) and FPKM values for assembled transcripts (Cufflinks text output files) are available in the NCBI Gene Expression Omnibus (Series GSE67894).

\section{Additional files}

Additional file 1: Figure S1. Gene coverage and comparison of D. immitis biological tissue replicas. (PDF $25 \mathrm{~kb}$ )

Additional file 2: Dataset S1. Transcript FPKM values for Dirofilaria immitis tissue transcriptome. (XLSX $369 \mathrm{~kb}$ )

Additional file 3: Table S1. List of over-represented GO terms and their associated $p$-values for tissue-associated $D$. immitis genes detected by either transcriptomics (red) or proteomics (blue). (XLSX $984 \mathrm{~kb}$ )

Additional file 4: Table S2. List of over-represented GO terms and their associated $\mathrm{p}$-values for tissue-associated $\mathrm{D}$. immitis genes that were also over-represented in the transcriptome of either adult male (AM) or adult female (AF) D. immitis. GO terms identified by transcriptomic analysis are highlighted in red while $\mathrm{GO}$ terms identified by proteomic analysis are in blue. (XLSX $55 \mathrm{~kb}$ )

Additional file 5: Figure S2. Significance ( $p$-value) of $G O$ terms shared between D. immitis adult male and testestranscriptomes. (PDF $43 \mathrm{~kb}$ )

Additional file 6: Figure S3. Clustered transcriptomic data of Wolbachia gene expression profiles in D. immitis tissues. (PDF $21 \mathrm{~kb}$ )

Additional file 7: Table S3. D. immitis tissue-associated Wolbachia transcript list based on hierarchical clustering. (XLSX $226 \mathrm{~kb}$ )

Additional file 8: Table S4. Tissue-associated expression and proteomic detection of the 834 D. immitis genes uniquely shared with B. malayi. (XLSX $170 \mathrm{~kb})$

Additional file 9: Table S5. List of D. immitis genes that are significantly $(q<0.01)$ differentially expressed between various $D$. immitis tissues. (XLSX $998 \mathrm{~kb})$

Additional file 10: Table S6. List of Wolbachia genes that are significantly $(q<0.01)$ differentially expressed between various $D$. immitis tissues. (XLSX $87 \mathrm{~kb}$ )

Additional file 11: Dataset S2. Dirofilaria immitis and Wolbachia proteins identified in D. immitis tissues. (XLSX $58 \mathrm{~kb}$ )

Additional file 12: Table S7. Gene IDs, peptide spectral counts and protein descriptions for all $4162 \mathrm{D}$. immitis proteins detected by mass spectrometry in D. immitis tissues. (XLSX $851 \mathrm{~kb}$ )

Additional file 13: Figure S4. Clustered proteomic data (peptide spectral matches) of D. immitis proteins detected inD. immitis tissues. (PDF $50 \mathrm{~kb}$ )

Additional file 14: Table S8. Gene IDs, peptide spectral counts and protein descriptions for all 170 Wolbachia proteins detected by mass spectrometry in D. immitis tissues. (XLSX $56 \mathrm{~kb}$ )

Additional file 15: Figure S5. Euler diagrams illustrating D. immitis genes detected by transcriptomic (green) and proteomic (blue) methods in various female D. immitis tissues: (A) body wall, (B) uterus, (C) head, (D) intestine. (PDF $1275 \mathrm{~kb}$ )

Additional file 16: Figure S6. Euler diagrams illustrating D. immitis genes detected by transcriptomic and proteomicmethods. (PDF $89 \mathrm{~kb}$ )

\section{Abbreviations}

AF: Adult female; AM: Adult male; FASP: Filter aided sample preparation: FBW: Female body wall; FDR: False discovery rate; FH: Female head;
FI: Female intestine; FPKM: Fragments Per Kilobase of transcript per Million mapped reads; FU: Female uterus; GO: Gene ontology; LC-MS/MS: Liquid chromatography-tandem mass spectrometry; MBW: Male body wall; MH: Male head; MI: Male intestine; MS: Male spicule; MS/MS: Tandem mass spectrometry; msp: Major sperm protein; MT: Male testis; RP: Reverse phase; SCX: Strong cation exchange; T4SS: Type 4 secretion system; wBm: Wolbachia from Brugia malayi; wDi: Wolbachia from Dirofilaria immitis; wOo: Wolbachia from Onchocerca ochengi.

\section{Competing interests}

The authors declare that they have no competing interests.

\section{Authors' contributions}

Conceived and designed the experiments: ANL NCV JMF BES MLM. Performed the experiments and analyzed the data: ANL KGA CMM NCV MLM. Drafted the manuscript: ANL. All authors read, edited and approved the final manuscript.

\section{Acknowledgements}

We wish to thank Timothy Anderson for the development of worm dissection methods, the NEB DNA Sequencing Core for sequencing of transcriptomic samples, Dr. Jack Benner of the NEB Mass Spectrometry lab for preliminary sample analysis, Dr. Manesh Shah (University of Tennessee, Knoxville) and Dr. Laurence Ettwiller for bioinformatic support. We also wish to thank Dr. Francine Perler for critical reading of the manuscript and Dr. Donald Comb, Dr. William Jack, Dr. Clotilde Carlow and James Ellard for their continued scientific support. This work was funded by New England Biolabs, Inc.

\section{Author details}

${ }^{1}$ Genome Biology Division, New England Biolabs, Inc., 240 County Road, Ipswich, MA 01938, USA. '2Department of Biology and Microbiology, University of Wisconsin Oshkosh, Oshkosh, WI 54901, USA. ${ }^{3}$ Chemical Biology Division, New England Biolabs, Inc., 240 County Road, Ipswich, MA 01938, USA.

Received: 16 April 2015 Accepted: 15 October 2015

Published online: 11 November 2015

\section{References}

1. Taylor MJ, Hoerauf A, Bockarie M. Lymphatic filariasis and onchocerciasis. Lancet. 2010;376(9747):1175-85.

2. World Health Organization. GPELF Progress Report 2000-2009 and Strategic Plan 2010-2020. WHO/HTM/NTD/PCT/2010. Geneva: WHO; 2010

3. Taylor MJ, Awadzi K, Basanez MG, Biritwum N, Boakye D, Boatin B, et al. Onchocerciasis control: vision for the future from a Ghanian perspective. Parasit Vectors. 2009;2(1):7.

4. Osei-Atweneboana MY, Awadzi K, Attah SK, Boakye DA, Gyapong JO, Prichard RK. Phenotypic evidence of emerging ivermectin resistance in Onchocerca volvulus. PLoS Negl Trop Dis. 2011;5(3):e998.

5. Landmann F, Foster JM, Slatko B, Sullivan W. Asymmetric Wolbachia segregation during early Brugia malayi embryogenesis determines its distribution in adult host tissues. PLoS Negl Trop Dis. 2010;4(7):e758.

6. Taylor MJ, Bandi C, Hoerauf A. Wolbachia bacterial endosymbionts of filarial nematodes. Adv Parasitol. 2005:60:245-84.

7. Bandi C, Trees AJ, Brattig NW. Wolbachia in filarial nematodes: evolutionary aspects and implications for the pathogenesis and treatment of filarial diseases. Vet Parasitol. 2001;98(1-3):215-38.

8. Hoerauf A, Mand S, Adjei O, Fleischer B, Buttner DW. Depletion of Wolbachia endobacteria in Onchocerca volvulus by doxycycline and microfilaridermia after ivermectin treatment. Lancet. 2001;357(9266):1415-6.

9. Hoerauf A, Specht S, Buttner M, Pfarr K, Mand S, Fimmers R, et al. Wolbachia endobacteria depletion by doxycycline as antifilarial therapy has macrofilaricidal activity in onchocerciasis: a randomized placebo-controlled study. Med Microbiol Immunol. 2008;197(3):295-311.

10. Supali T, Djuardi Y, Pfarr KM, Wibowo H, Taylor MJ, Hoerauf A, et al. Doxycycline treatment of Brugia malayi-infected persons reduces microfilaremia and adverse reactions after diethylcarbamazine and albendazole treatment. Clin Infect Dis. 2008;46(9):1385-93. 
11. Foster J, Ganatra M, Kamal I, Ware J, Makarova K, Ivanova N, et al. The Wolbachia genome of Brugia malayi: endosymbiont evolution within a human pathogenic nematode. PLoS Biol. 2005;3(4):e121.

12. Dunning Hotopp JC, Lin M, Madupu R, Crabtree J, Angiuoli SV, Eisen JA, et al. Comparative genomics of emerging human ehrlichiosis agents. PLoS Genet. 2006;2(2):e21.

13. Brownlie JC, Adamski M, Slatko B, McGraw EA. Diversifying selection and host adaptation in two endosymbiont genomes. BMC Evol Biol. 2007;7:68.

14. Godel C, Kumar S, Koutsovoulos G, Ludin P, Nilsson D, Comandatore F, et al. The genome of the heartworm, Dirofilaria immitis, reveals drug and vaccine targets. Faseb J. 2012;26(11):4650-61.

15. Choi YJ, Ghedin E, Berriman M, McQuillan J, Holroyd N, Mayhew GF, et al. A deep sequencing approach to comparatively analyze the transcriptome of lifecycle stages of the filarial worm, Brugia malayi. PLoS Negl Trop Dis. 2011;5(12):e1409.

16. Luck AN, Evans CC, Riggs MD, Foster JM, Moorhead AR, Slatko BE, et al. Concurrent transcriptional profiling of Dirofilaria immitis and its Wolbachia endosymbiont throughout the nematode life cycle reveals coordinated gene expression. BMC Genomics. 2014;15(1):1041.

17. Rosa BA, Jasmer DP, Mitreva M. Genome-wide tissue-specific gene expression, co-expression and regulation of co-expressed genes in adult nematode Ascaris suum. PLoS Negl Trop Dis. 2014;8(2):e2678.

18. Giardine B, Riemer C, Hardison RC, Burhans R, Elnitski L, Shah P, et al. Galaxy: a platform for interactive large-scale genome analysis. Genome Res. 2005;15(10):1451-5.

19. Blankenberg D, Von Kuster G, Coraor N, Ananda G, Lazarus R, Mangan M Nekrutenko A, Taylor J. Galaxy: a web-based genome analysis tool for experimentalists. Curr Protoc Mol Biol..2010;4:19:Unit 19.10.1121.doi.10.1002/0471142727.mb1910s89

20. Goecks J, Nekrutenko A, Taylor J. Galaxy: a comprehensive approach for supporting accessible, reproducible, and transparent computational research in the life sciences. Genome Biol. 2010;11(8):R86.

21. Andrews S. FastQC: a quality control tool for high throughput sequence data. 2010. http://www.bioinformatics.babraham.ac.uk/projects/fastqc.

22. Trapnell C, Williams BA, Pertea G, Mortazavi A, Kwan G, van Baren MJ, et al. Transcript assembly and quantification by RNA-Seq reveals unannotated transcripts and isoform switching during cell differentiation. Nat Biotechnol. 2010;28(5):511-5

23. Trapnell C, Pachter L, Salzberg SL. TopHat: discovering splice junctions with RNA-Seq. Bioinformatics. 2009;25(9):1105-11.

24. Langmead B, Trapnell C, Pop M, Salzberg SL. Ultrafast and memory-efficient alignment of short DNA sequences to the human genome. Genome Biol. 2009;10(3):R25

25. Eisen MB, Spellman PT, Brown PO, Botstein D. Cluster analysis and display of genome-wide expression patterns. Proc Natl Acad Sci U S A. 1998;95(25):14863-8.

26. Zheng Q, Wang XJ. GOEAST: a web-based software toolkit for Gene Ontology enrichment analysis. Nucleic Acids Res. 2008;36(Web Server issue):W358-363.

27. Schleicher TR, VerBerkmoes NC, Shah M, Nyholm SV. Colonization state influences the hemocyte proteome in a beneficial squid-Vibrio symbiosis. Mol Cell Proteomics. 2014;13(10):2673-86.

28. Darby C, Cosma CL, Thomas JH, Manoil C. Lethal paralysis of Caenorhabditis elegans by Pseudomonas aeruginosa. Proc Natl Acad Sci U S A. 1999;96(26):15202-7.

29. Waterston RH, Thomson JN, Brenner S. Mutants with altered muscle structure of Caenorhabditis elegans. Dev Biol. 1980;77(2):271-302.

30. Moerman DG, Benian GM, Barstead RJ, Schriefer LA, Waterston RH. Identification and intracellular localization of the unc-22 gene product of Caenorhabditis elegans. Genes Dev. 1988;2(1):93-105.

31. Vogel BE, Hedgecock EM. Hemicentin, a conserved extracellular member of the immunoglobulin superfamily, organizes epithelial and other cell attachments into oriented line-shaped junctions. Development. 2001;128(6):883-94.

32. Vogel BE, Muriel JM, Dong C, Xu X. Hemicentins: what have we learned from worms? Cell Res. 2006;16(11):872-8.

33. Avery L. The genetics of feeding in Caenorhabditis elegans. Genetics. 1993;133(4):897-917.

34. Raizen DM, Lee RY, Avery L. Interacting genes required for pharyngea excitation by motor neuron MC in Caenorhabditis elegans. Genetics. 1995;141(4):1365-82.
35. Van Gilst MR, Hadjivassiliou H, Jolly A, Yamamoto KR. Nuclear hormone receptor NHR-49 controls fat consumption and fatty acid composition in C. elegans. PLoS Biol. 2005;3(2):e53.

36. Van Gilst MR, Hadjivassiliou H, Yamamoto KR. A Caenorhabditis elegans nutrient response system partially dependent on nuclear receptor NHR-49. Proc Natl Acad Sci U S A. 2005;102(38):13496-501.

37. Hahn-Windgassen A, Van Gilst MR. The Caenorhabditis elegans HNF4alpha Homolog, NHR-31, mediates excretory tube growth and function through coordinate regulation of the vacuolar ATPase. PLoS Genet. 2009;5(7):e1000553.

38. Coleman DL, Eicher EM. Fat (fat) and tubby (tub): two autosomal recessive mutations causing obesity syndromes in the mouse. J Hered. 1990;81(6):424-7.

39. Mukhopadhyay A, Deplancke B, Walhout AJ, Tissenbaum HA. C. elegans tubby regulates life span and fat storage by two independent mechanisms. Cell Metab. 2005;2(1):35-42.

40. Huang CC, Hall DH, Hedgecock EM, Kao G, Karantza V, Vogel BE, et al. Laminin alpha subunits and their role in C. elegans development. Development. 2003;130(14):3343-58.

41. Darby AC, Armstrong SD, Bah GS, Kaur G, Hughes MA, Kay SM, et al. Analysis of gene expression from the Wolbachia genome of a filarial nematode supports both metabolic and defensive roles within the symbiosis. Genome Res. 2012;22(12):2467-77.

42. Armstrong SD, Babayan SA, Lhermitte-Vallarino N, Gray N, Xia D, Martin C, et al. Comparative Analysis of the Secretome from a Model Filarial Nematode (Litomosoides sigmodontis) Reveals Maximal Diversity in Gravid Female Parasites. Mol Cell Proteomics. 2014;13(10):2527-44.

43. Prusse A, Vollmer S, Diesfeld HJ. Immunocytochemical and ultrastructural studies on Dipetalonema viteae (Filarioidea). J Helminthol. 1983;57(2):127-42.

44. Kostrouchova M, Krause M, Kostrouch Z, Rall JE. CHR3: a Caenorhabditis elegans orphan nuclear hormone receptor required for proper epidermal development and molting. Development. 1998;125(9):1617-26.

45. Kouns NA, Nakielna J, Behensky F, Krause MW, Kostrouch Z, Kostrouchova M. NHR-23 dependent collagen and hedgehog-related genes required for molting. Biochem Biophys Res Commun. 2011;413(4):515-20.

46. Kostrouchova M, Krause M, Kostrouch Z, Rall JE. Nuclear hormone receptor CHR3 is a critical regulator of all four larval molts of the nematode Caenorhabditis elegans. Proc Natl Acad Sci U S A. 2001;98(13):7360-5.

47. Starich TA, Herman RK, Shaw JE. Molecular and genetic analysis of unc-7, a Caenorhabditis elegans gene required for coordinated locomotion. Genetics. 1993;133(3):527-41.

48. Barnes TM, Hekimi S. The Caenorhabditis elegans avermectin resistance and anesthetic response gene unc- 9 encodes a member of a protein family implicated in electrical coupling of excitable cells. J Neurochem. 1997;69(6):2251-60.

49. Van Auken K, Weaver D, Robertson B, Sundaram M, Saldi T, Edgar L, et al. Roles of the Homothorax/Meis/Prep homolog UNC-62 and the Exd/Pbx homologs CEH-20 and CEH-40 in i embryogenesis. Development. 2002;129(22):5255-68.

50. Roayaie K, Crump JG, Sagasti A, Bargmann Cl. The G alpha protein ODR-3 mediates olfactory and nociceptive function and controls cilium morphogenesis in C. elegans olfactory neurons. Neuron. 1998;20(1):55-67.

51. Crossgrove K, Maina CV, Robinson-Rechavi M, Lochner MC. Orthologues of the Drosophila melanogaster E75 molting control gene in the filarial parasites Brugia malayi and Dirofilaria immitis. Mol Biochem Parasitol. 2008;157(1):92-7.

52. Mimoto A, Fujii M, Usami M, Shimamura M, Hirabayashi N, Kaneko T, et al. Identification of an estrogenic hormone receptor in Caenorhabditis elegans. Biochem Biophys Res Commun. 2007:364(4):883-8.

53. Kwon JY, Kim-Ha J, Lee BJ, Lee J. The MED-7 transcriptional mediato encoded by let-49 is required for gonad and germ cell development in Caenorhabditis elegans. FEBS Lett. 2001;508(3):305-8.

54. Chen L, Ong B, Bennett V. LAD-1, the Caenorhabditis elegans L1CAM homologue, participates in embryonic and gonadal morphogenesis and is a substrate for fibroblast growth factor receptor pathway-dependent phosphotyrosine-based signaling. J Cell Biol. 2001;154(4):841-55.

55. Wen C, Levitan D, Li X. Greenwald I: spr-2, a suppressor of the egg-laying defect caused by loss of sel-12 presenilin in Caenorhabditis elegans, is a member of the SET protein subfamily. Proc Natl Acad Sci U S A. 2000;97(26):14524-9.

56. Unhavaithaya Y, Shin TH, Miliaras N, Lee J, Oyama T, Mello CC. MEP-1 and a homolog of the NURD complex component Mi-2 act together to maintain germline-soma distinctions in C. elegans. Cell. 2002;111(7):991-1002. 
57. Karashima T, Sugimoto A, Yamamoto M. Caenorhabditis elegans homologue of the human azoospermia factor DAZ is required for oogenesis but not for spermatogenesis. Development. 2000;127(5):1069-79.

58. Zhang Y, Foster JM, Nelson LS, Ma D, Carlow CK. The chitin synthase genes chs-1 and chs-2 are essential for C. elegans development and responsible for chitin deposition in the eggshell and pharynx, respectively. Dev Biol. 2005;285(2):330-9.

59. Choy RK, Thomas JH. Fluoxetine-resistant mutants in C. elegans define a novel family of transmembrane proteins. Mol Cell. 1999;4(2):143-52.

60. Heid PJ, Raich WB, Smith R, Mohler WA, Simokat K, Gendreau SB, et al. The zinc finger protein DIE-1 is required for late events during epithelial cell rearrangement in C. elegans. Dev Biol. 2001;236(1):165-80.

61. Tsuboi D, Qadota H, Kasuya K, Amano M, Kaibuchi K. Isolation of the interacting molecules with GEX-3 by a novel functional screening. Biochem Biophys Res Commun. 2002;292(3):697-701.

62. Kumar S, Chaudhary K, Foster JM, Novelli JF, Zhang Y, Wang S, et al. Mining predicted essential genes of Brugia malayi for nematode drug targets. PLoS One. 2007;2(11):e1189.

63. Ellis RE, Kimble J. The fog-3 gene and regulation of cell fate in the germ line of Caenorhabditis elegans. Genetics. 1995;139(2):561-77.

64. Suh $N$, Jedamzik B, Eckmann CR, Wickens M, Kimble J. The GLD-2 poly(A) polymerase activates gld-1 mRNA in the Caenorhabditis elegans germ line. Proc Natl Acad Sci U S A. 2006;103(41):15108-12.

65. L'Hernault SW, Shakes DC, Ward S. Developmental genetics of chromosome I spermatogenesis-defective mutants in the nematode Caenorhabditis elegans. Genetics. 1988;120(2):435-52.

66. Arduengo PM, Appleberry OK, Chuang P, L'Hernault SW. The presenilin protein family member SPE-4 localizes to an ER/Golgi derived organelle and is required for proper cytoplasmic partitioning during Caenorhabditis elegans spermatogenesis. J Cell Sci. 1998;111(Pt 24):3645-54.

67. Singson A, Mercer KB, L'Hernault SW. The C. elegans spe-9 gene encodes a sperm transmembrane protein that contains EGF-like repeats and is required for fertilization. Cell. 1998;93(1):71-9.

68. Zugasti O, Rajan J, Kuwabara PE. The function and expansion of the Patched- and Hedgehog-related homologs in C. elegans. Genome Res. 2005;15(10):1402-10.

69. Lim HH, Park BJ, Choi HS, Park CS, Eom SH, Ahnn J. Identification and characterization of a putative $C$. elegans potassium channel gene (Ce-slo-2) distantly related to $\mathrm{Ca}(2+)$-activated $\mathrm{K}(+)$ channels. Gene. 1999;240(1):35-43.

70. Liu P, Ge Q, Chen B, Salkoff L, Kotlikoff MI, Wang ZW. Genetic dissection of ion currents underlying all-or-none action potentials in C. elegans body-wall muscle cells. J Physiol. 2011;589(Pt 1):101-17.

71. Smith WD. Protection in lambs immunised with Haemonchus contortus gut membrane proteins. Res Vet Sci. 1993;54(1):94-101.

72. Cachat E, Newlands GF, Ekoja SE, McAllister H, Smith WD. Attempts to immunize sheep against Haemonchus contortus using a cocktail of recombinant proteases derived from the protective antigen, $\mathrm{H}$-gal-GP. Parasite Immunol. 2010;32(6):414-9.

73. McGonigle S, Yoho ER, James ER. Immunisation of mice with fractions derived from the intestines of Dirofilaria immitis. Int J Parasitol. 2001;31(13):1459-66.

74. Lapierre LR, De Magalhaes Filho CD, McQuary PR, Chu CC, Visvikis O, Chang JT, et al. The TFEB orthologue HLH-30 regulates autophagy and modulates longevity in Caenorhabditis elegans. Nat Commun. 2013:4:2267.

75. Uno M, Honjoh S, Matsuda M, Hoshikawa H, Kishimoto S, Yamamoto T, et al. A fasting-responsive signaling pathway that extends life span in $C$. elegans. Cell Rep. 2013;3(1):79-91.

76. Mango SE, Lambie EJ, Kimble J. The pha-4 gene is required to generate the pharyngeal primordium of Caenorhabditis elegans. Development. 1994;120(10):3019-31.

77. Taubert S, Van Gilst MR, Hansen M, Yamamoto KR. A Mediator subunit, MDT-15, integrates regulation of fatty acid metabolism by NHR-49dependent and -independent pathways in C. elegans. Genes Dev. 2006;20(9):1137-49.

78. Watts JL, Browse J. Isolation and characterization of a Delta 5-fatty acid desaturase from Caenorhabditis elegans. Arch Biochem Biophys. 1999;362(1):175-82.

79. Picken NC, Eschenlauer S, Taylor P, Page AP, Walkinshaw MD. Structural and biological characterisation of the gut-associated cyclophilin B isoforms from Caenorhabditis elegans. J Mol Biol. 2002;322(1):15-25.
80. Joshua GW. Functional analysis of leucine aminopeptidase in Caenorhabditis elegans. Mol Biochem Parasitol. 2001;113(2):223-32.

81. Korolnek T, Zhang J, Beardsley S, Scheffer GL, Hamza I. Control of metazoan heme homeostasis by a conserved multidrug resistance protein. Cell Metab. 2014;19(6):1008-19.

82. Davis MW, Fleischhauer $\mathrm{R}$, Dent JA, Joho RH, Avery L. A mutation in the C. elegans EXP-2 potassium channel that alters feeding behavior. Science. 1999;286(5449):2501-4

83. Mohrlen F, Hutter H, Zwilling R. The astacin protein family in Caenorhabditis elegans. Eur J Biochem. 2003;270(24):4909-20.

84. Baylis HA, Furuichi T, Yoshikawa F, Mikoshiba K, Sattelle DB. Inositol 1,4,5trisphosphate receptors are strongly expressed in the nervous system, pharynx, intestine, gonad and excretory cell of Caenorhabditis elegans and are encoded by a single gene (itr-1). J Mol Biol. 1999;294(2):467-76.

85. Walker DS, Gower NJ, Ly S, Bradley GL, Baylis HA. Regulated disruption of inositol 1,4,5-trisphosphate signaling in Caenorhabditis elegans reveals new functions in feeding and embryogenesis. Mol Biol Cell. 2002;13(4):1329-37.

86. Han HF, Beckerle MC. The ALP-Enigma protein ALP-1 functions in actin filament organization to promote muscle structural integrity in Caenorhabditis elegans. Mol Biol Cell. 2009;20(9):2361-70.

87. Goetinck S, Waterston RH. The Caenorhabditis elegans muscle-affecting gene unc-87 encodes a novel thin filament-associated protein. J Cell Biol. 1994;127(1):79-93.

88. Goetinck S, Waterston RH. The Caenorhabditis elegans UNC-87 protein is essential for maintenance, but not assembly, of bodywall muscle. J Cell Biol. 1994;127(1):71-8.

89. Kranewitter WJ, Ylanne J, Gimona M. UNC-87 is an actin-bundling protein. J Biol Chem. 2001;276(9):6306-12.

90. Barstead RJ, Waterston RH. The basal component of the nematode densebody is vinculin. J Biol Chem. 1989;264(17):10177-85.

91. Ono K, Yu R, Mohri K, Ono S. Caenorhabditis elegans kettin, a large immunoglobulin-like repeat protein, binds to filamentous actin and provides mechanical stability to the contractile apparatuses in body wall muscle. Mol Biol Cell. 2006;17(6):2722-34.

92. Forbes JG, Flaherty DB, Ma K, Qadota H, Benian GM, Wang K. Extensive and modular intrinsically disordered segments in C. elegans TTN-1 and implications in filament binding, elasticity and oblique striation. J Mol Biol. 2010;398(5):672-89.

93. Hiatt SM, Duren HM, Shyu YJ, Ellis RE, Hisamoto N, Matsumoto K, et al. Caenorhabditis elegans FOS-1 and JUN-1 regulate plc-1 expression in the spermatheca to control ovulation. Mol Biol Cell. 2009;20(17):3888-95.

94. Broday L, Kolotuev I, Didier C, Bhoumik A, Podbilewicz B, Ronai Z. The LIM domain protein UNC-95 is required for the assembly of muscle attachment structures and is regulated by the RING finger protein RNF-5 in C. elegans. J Cell Biol. 2004;165(6):857-67.

95. Bennuru S, Meng Z, Ribeiro JM, Semnani RT, Ghedin E, Chan K, et al. Stagespecific proteomic expression patterns of the human filarial parasite Brugia malayi and its endosymbiont Wolbachia. Proc Natl Acad Sci U S A. 2011;108(23):9649-54.

96. McKay SJ, Johnsen R, Khattra J, Asano J, Baillie DL, Chan S, et al. Gene expression profiling of cells, tissues, and developmental stages of the nematode C. elegans. Cold Spring Harb Symp Quant Biol. 2003;68:159-69.

97. Chen B, Liu P, Wang SJ, Ge Q, Zhan H, Mohler WA, et al. alpha-Catulin CTN1 is required for BK channel subcellular localization in C. elegans body-wall muscle cells. Embo J. 2010;29(18):3184-95.

\section{Submit your next manuscript to BioMed Central and take full advantage of:}

- Convenient online submission

- Thorough peer review

- No space constraints or color figure charges

- Immediate publication on acceptance

- Inclusion in PubMed, CAS, Scopus and Google Scholar

- Research which is freely available for redistribution 